\title{
Boundedness and compactness of a new product-type operator from a general space to Bloch-type spaces
}

\author{
Stevo Stević ${ }^{1,2^{*}}$, Ajay K Sharma ${ }^{3}$ and Ram Krishan ${ }^{3}$
}

\author{
"Correspondence: sstevic@ptt.rs \\ 'Mathematical Institute of the \\ Serbian Academy of Sciences, Knez \\ Mihailova 36/III, Beograd, 11000, \\ Serbia \\ ${ }^{2}$ Operator Theory and Applications \\ Research Group, Department of \\ Mathematics, King Abdulaziz \\ University, P.O. Box 80203, Jeddah, \\ 21589, Saudi Arabia \\ Full list of author information is \\ available at the end of the article
}

\begin{abstract}
We characterize the boundedness and compactness of a product-type operator, which, among others, includes all the products of the single composition, multiplication, and differentiation operators, from a general space to Bloch-type spaces. We also give some upper and lower bounds for the norm of the operator.
\end{abstract}

MSC: Primary 47B38; secondary 46E15

Keywords: product-type operator; Bloch-type space; boundedness; compactness

\section{Introduction}

Let $\mathbb{D}$ be the open unit disk in the complex plane $\mathbb{C}, \partial \mathbb{D}$ its boundary, $d A(z)$ the normalized area measure on $\mathbb{D}$ (i.e., $A(\mathbb{D})=1$ ), $H(\mathbb{D})$ the class of all holomorphic functions on $\mathbb{D}$, and $S(\mathbb{D})$ the family of all holomorphic self-maps of $\mathbb{D}$. Let

$$
\eta_{a}(z)=\frac{a-z}{1-\bar{a} z}, \quad a, z \in \mathbb{D},
$$

that is, the involutive automorphism of $\mathbb{D}$ interchanging points $a$ and 0 . Simple calculation shows that

$$
\frac{1-\left|\eta_{a}(z)\right|^{2}}{1-|z|^{2}}=\frac{1-|a|^{2}}{|1-\bar{a} z|^{2}}=\left|\eta_{a}^{\prime}(z)\right| .
$$

A strictly positive continuous function $\mu$ on $\mathbb{D}$ is called weight. A weight $\mu$ is called radial if $\mu(z)=\mu(|z|)$ for every $z \in \mathbb{D}$. A radial weight $\mu$ is called typical if it is nonincreasing with respect to $|z|$ and $\mu(z) \rightarrow 0$ as $|z| \rightarrow 1$. For a weight $\mu$, the Bloch-type space $\mathcal{B}_{\mu}=\mathcal{B}_{\mu}(\mathbb{D})$ is the space of all $f \in H(\mathbb{D})$ such that

$$
b_{\mu}(f)=\sup _{z \in \mathbb{D}} \mu(z)\left|f^{\prime}(z)\right|<\infty
$$

The little Bloch-type space $\mathcal{B}_{\mu, 0}=\mathcal{B}_{\mu, 0}(\mathbb{D})$ consists of all $f \in \mathcal{B}_{\mu}$ such that

$$
\lim _{|z| \rightarrow 1} \mu(z)\left|f^{\prime}(z)\right|=0 .
$$

(c) 2016 Stević et al. This article is distributed under the terms of the Creative Commons Attribution 4.0 International License (http://creativecommons.org/licenses/by/4.0/), which permits unrestricted use, distribution, and reproduction in any medium, provided you give appropriate credit to the original author(s) and the source, provide a link to the Creative Commons license, and indicate if changes were made. 
The space $\mathcal{B}_{\mu}$ is a Banach space with the norm

$$
\|f\|_{\mathcal{B}_{\mu}}=|f(0)|+b_{\mu}(f)
$$

and if $\mu$ is a typical weight, then $\mathcal{B}_{\mu, 0}$ is a closed subspace of $\mathcal{B}_{\mu}$. When $\mu(z)=\left(1-|z|^{2}\right)^{\alpha}$, $\alpha>0, \mathcal{B}_{\mu}$ reduces to the $\alpha$-Bloch space, denoted by $\mathcal{B}^{\alpha}$, whereas $\mathcal{B}_{\mu, 0}$ reduces to the little $\alpha$-Bloch space $\mathcal{B}_{0}^{\alpha}$. For some information on Bloch-type spaces, see, for example, [1, 2].

Likewise, for a weight $\mu$, the weighted-type space $H_{\mu}^{\infty}=H_{\mu}^{\infty}(\mathbb{D})$ consists of all $f \in H(\mathbb{D})$ such that

$$
\sup _{z \in \mathbb{D}} \mu(z)|f(z)|<\infty
$$

and the little weighted-type space $H_{\mu, 0}^{\infty}=H_{\mu, 0}^{\infty}(\mathbb{D})$ consists of all $f \in H_{\mu}^{\infty}$ such that

$$
\lim _{|z| \rightarrow 1} \mu(z)|f(z)|=0
$$

(see, e.g., [3]).

For $0<p<\infty,-2<q<\infty$, and $0 \leq s<\infty$, the spaces $F(p, q, s)$ and $F_{0}(p, q, s)$ are defined as the sets of all $f \in H(\mathbb{D})$ such that

$$
M(f)=\left(\sup _{a \in \mathbb{D}} \int_{\mathbb{D}}\left|f^{\prime}(z)\right|^{p}\left(1-|z|^{2}\right)^{q}\left(1-\left|\eta_{a}(z)\right|^{2}\right)^{s} d A(z)\right)^{1 / p}<\infty
$$

and such that

$$
\lim _{|a| \rightarrow 1} \int_{\mathbb{D}}\left|f^{\prime}(z)\right|^{p}\left(1-|z|^{2}\right)^{q}\left(1-\left|\eta_{a}(z)\right|^{2}\right)^{s} d A(z)=0
$$

for $0<s<\infty$, respectively, whereas if $s=0$, then $F(p, q, 0)=F_{0}(p, q, 0)$ is defined naturally as the set of all $f \in H(\mathbb{D})$ such that

$$
M(f)=\left(\int_{\mathbb{D}}\left|f^{\prime}(z)\right|^{p}\left(1-|z|^{2}\right)^{q} d A(z)\right)^{1 / p}<\infty
$$

The spaces $F(p, q, s)$ and $F_{0}(p, q, s)$ are known as general families of function spaces. For $1 \leq p<\infty, F(p, q, s)$ is a Banach space with respect to the norm

$$
\|f\|_{F(p, q, s)}=|f(0)|+M(f)
$$

and $F_{0}(p, q, s)$ is a closed subspace of $F(p, q, s)$. The importance of these spaces stems from the fact that for appropriate parameter values of $p, q$, and $s$, they coincide with several classical function spaces. For example, $F(2,1,0)$ is the Hardy space $H^{2}, F(p, p+\alpha, 0)$, $\alpha>-1$, is the weighted Bergman space $A_{\alpha}^{p}, F(p, q, s)=\mathcal{B}^{\frac{2+q}{p}}$ and $F_{0}(p, q, s)=\mathcal{B}_{0}^{\frac{2+q}{p}}$ for $s>1$, $F(p, q, s) \subset \mathcal{B}^{\frac{2+q}{p}}$ and $F_{0}(p, q, s) \subset \mathcal{B}_{0}^{\frac{2+q}{p}}$ for $0<s \leq 1, F(2,0, p)=Q_{p}$ and $F_{0}(2,0, p)=Q_{p, 0}$, and $F(2,0,1)=B M O A$, the space of analytic functions with bounded mean oscillation, and $F_{0}(2,0,1)=V M O A$, the space of analytic functions with vanishing mean oscillation. 
If $q+s \leq-1, F(p, q, s)$ is trivial, that is, equal to the space of constant functions, whereas for $q+s>-1$, it is nontrivial [4].

Let $\varphi \in S(\mathbb{D})$. The composition operator $C_{\varphi}$ induced by $\varphi$ is defined by

$$
C_{\varphi} f=f \circ \varphi, \quad f \in H(\mathbb{D}) .
$$

For $\psi \in H(\mathbb{D})$, the multiplication operator $M_{\psi}$ is defined on $H(\mathbb{D})$ by

$$
M_{\psi} f(z)=\psi(z) f(z), \quad f \in H(\mathbb{D}) .
$$

The following product of these two operators $W_{\varphi, \psi}=M_{\psi} \circ C_{\varphi}$, the so-called weighted composition operator, has been studied a lot recently.

The differentiation operator denoted by $D$ is defined by

$$
D f=f^{\prime}, \quad f \in H(\mathbb{D}) .
$$

At first, the experts studied operator-theoretic properties of these operators on spaces of holomorphic functions in terms of their symbols separately. A systematic study of products of concrete linear operators between spaces of holomorphic functions started approximately a decade ago; see, for example, [5-32], and the related references therein. The first product-type operators different from weighted composition operators, which have been considerably studied, are the products of composition and differentiation operators (see, e.g., $[6,7,9,10,13,15,16,20,25,28]$ and the references therein). Quite recently, there appeared some more complex product-type operators that include some of classical operators, such as composition, differentiation, multiplication, or integral-type operators (see, e.g., $[17-19,28,30,33-35]$ and the related references therein).

The product-type operators consisting of exactly one composition, multiplication, and differentiation operator are the following:

$$
\begin{aligned}
& \left(M_{\psi} C_{\varphi} D f\right)(z)=\psi(z) f^{\prime}(\varphi(z)), \\
& \left(M_{\psi} D C_{\varphi} f\right)(z)=\psi(z) \varphi^{\prime}(z) f^{\prime}(\varphi(z)), \\
& \left(C_{\varphi} M_{\psi} D f\right)(z)=\psi(\varphi(z)) f^{\prime}(\varphi(z)), \\
& \left(D M_{\psi} C_{\varphi} f\right)(z)=\psi^{\prime}(z) f(\varphi(z))+\psi(z) \varphi^{\prime}(z) f^{\prime}(\varphi(z)), \\
& \left(C_{\varphi} D M_{\psi} f\right)(z)=\psi^{\prime}(\varphi(z)) f(\varphi(z))+\psi(\varphi(z)) f^{\prime}(\varphi(z)), \\
& \left(D C_{\varphi} M_{\psi} f\right)(z)=\psi^{\prime}(\varphi(z)) \varphi^{\prime}(z) f(\varphi(z))+\psi(\varphi(z)) \varphi^{\prime}(z) f^{\prime}(\varphi(z)),
\end{aligned}
$$

for $z \in \mathbb{D}$ and $f \in H(\mathbb{D})$.

To treat the operators in (1) in a unified manner, Stević et al. [23, 24] introduced a generalized operator and studied it on the weighted Bergman spaces. Motivated by that operator, here we introduce the operator

$$
T_{\psi_{1}, \psi_{2}, \varphi}^{n} f(z)=\psi_{1}(z) f^{(n)}(\varphi(z))+\psi_{2}(z) f^{(n+1)}(\varphi(z)), \quad f \in H(\mathbb{D}),
$$

where $\psi_{1}, \psi_{2} \in H(\mathbb{D}), \varphi \in S(\mathbb{D})$, and $n \in \mathbb{N}_{0}$. It is worth noticing that, among others, the composition operator, multiplication operator, differentiation operator, as well as all the 
products of composition, multiplication, and differentiation operators in (1), can be obtained from the operators $T_{\psi_{1}, \psi_{2}, \varphi}^{0}$ and $T_{\psi_{1}, \psi_{2}, \varphi}^{1}$ by fixing $\psi_{1}$ and $\psi_{2}$. More specifically, we have $C_{\varphi}=T_{1,0, \varphi}^{0}, M_{\psi}^{0}=T_{\psi, 0, z}^{0}, D=T_{1,0, z}^{1}=T_{0,1, z}^{0}, M_{\psi} C_{\varphi}=T_{\psi, 0, \varphi}^{0}, C_{\varphi} D=T_{1,0, \varphi}^{1}=T_{0,1, \varphi}^{0}$, $C_{\varphi} M_{\psi}=T_{\psi \circ \varphi, 0, \varphi}^{0}, D C_{\varphi}=T_{0, \varphi^{\prime}, \varphi}^{0}=T_{\varphi^{\prime}, 0, \varphi}^{1}, M_{\psi} D=T_{0, \psi, z}^{0}=T_{\psi, 0, z}^{1}, D M_{\psi}=T_{\psi^{\prime}, \psi, z}^{0}, M_{\psi} C_{\varphi} D=$ $T_{0, \psi, \varphi}^{0}=T_{\psi, 0, \varphi}^{1}, M_{\psi} D C_{\varphi} f=T_{0, \psi \varphi^{\prime}, \varphi}^{0}=T_{\psi \varphi^{\prime}, 0, \varphi^{\prime}}^{1}, C_{\varphi} M_{\psi} D=T_{0, \psi \circ \varphi, \varphi}^{0}=T_{\psi \circ \varphi, 0, \varphi}^{1}, D M_{\psi} C_{\varphi}=$ $T_{\psi^{\prime}, \psi \varphi^{\prime}, \varphi^{\prime}}^{0}, C_{\varphi} D M_{\psi}=T_{\psi^{\prime} \circ \varphi, \psi \circ \varphi, \varphi^{\prime}}^{0}, D C_{\varphi} M_{\psi}=T_{\left(\psi^{\prime} \circ \varphi\right) \varphi^{\prime},(\psi \circ \varphi) \varphi^{\prime}, \varphi^{\circ}}^{0}$ Note also that, for $\psi_{2} \equiv 0$, we obtain the weighted differentiation composition operator, which was studied, for example, in $[17-19,28,30]$.

Our aim here is to characterize the boundedness and compactness of the operator $T_{\psi_{1}, \psi_{2}, \varphi}^{n}$ from the $F(p, q, s)$ and $F_{0}(p, q, s)$ spaces to Bloch-type spaces. The paper can be regarded as a continuation of our line of investigations in [6, 7, 9, 10, 15-24, 34, 35].

Throughout this paper, constants are denoted by $C$, they are positive and not necessarily the same at each occurrence. The notation $A \asymp B$ means that $B \lesssim A \lesssim B$, where $A \lesssim B$ means that there is a positive constant $C$ such that $A \leq C B$. We also use the standard convention $\prod_{j=k}^{k-1} a_{j}=1$.

\section{Boundedness and compactness of $T_{\psi_{1}, \psi_{2}, \varphi}^{n}$}

In this section, we prove our main results. Namely, we characterize the boundedness and compactness of the operator $T_{\psi_{1}, \psi_{2}, \varphi}^{n}$ from the $F(p, q, s)$ and $F_{0}(p, q, s)$ spaces to Blochtype spaces. We also give some upper and lower bounds for the norm of $T_{\psi_{1}, \psi_{2}, \varphi}^{n}$ : $F(p, q, s)\left(\right.$ or $\left.F_{0}(p, q, s)\right) \rightarrow B_{\mu}$.

For this purpose, we need several lemmas. The next lemma can be found in [4].

Lemma 1 Let $0<p, s<\infty,-2<q<\infty, q+s>-1$, and $f \in F(p, q, s)$. Then

$$
\|f\|_{\mathcal{B}} \frac{2+q}{p} \lesssim\|f\|_{F(p, q, s)}
$$

Moreover, iff $\in F_{0}(p, q, s)$, then $f \in \mathcal{B}_{0}^{\frac{2+q}{p}}$.

The following folklore point-evaluation result can be found, for example, in [2].

Lemma 2 Let $\alpha>0$ and $f \in \mathcal{B}^{\alpha}$. Then

$$
|f(z)| \lesssim \begin{cases}\|f\|_{\mathcal{B}^{\alpha}}, & 0<\alpha<1 \\ \|f\|_{\mathcal{B}^{\alpha}} \ln \frac{2}{1-|z|^{2}}, & \alpha=1, \\ \frac{\|f\|_{\mathcal{B}^{\alpha}}}{\left(1-|z|^{2}\right)^{\alpha-1}}, & \alpha>1\end{cases}
$$

and

$$
\left|f^{(n)}(z)\right| \lesssim \frac{\|f\|_{\mathcal{B}^{\alpha}}}{\left(1-|z|^{2}\right)^{\alpha+n-1}}
$$

for each $n \in \mathbb{N}$.

The following lemma can easily be obtained by combining the inequalities in Lemmas 1 and 2 . 
Lemma 3 Let $0<p, s<\infty,-2<q<\infty, q+s>-1$, and $f \in F(p, q, s)$. Then

$$
|f(z)| \lesssim \begin{cases}\|f\|_{F(p, q, s)}, & p>q+2 \\ \|f\|_{F(p, q, s)} \ln \frac{2}{1-|z|^{2}}, & p=2+q \\ \frac{\|f\|_{F(p, q, s)}}{\left(1-|z|^{2}\right)^{\frac{2+q}{p}-1}}, & p<2+q\end{cases}
$$

and

$$
\left|f^{(n)}(z)\right| \lesssim \frac{\|f\|_{F(p, q, s)}}{\left(1-|z|^{2}\right)^{\frac{2+q}{p}+n-1}}, \quad n \in \mathbb{N} .
$$

The following lemma gives us important test functions belonging to the $F_{0}(p, q, s)$ space.

Lemma 4 Let $0<p, s<\infty,-2<q<\infty, q+s>-1$, and

$$
f_{w}(z)=\frac{1-|w|^{2}}{(1-\bar{w} z)^{\frac{2+q}{p}}}, \quad z, w \in \mathbb{D} .
$$

Then $\sup _{w \in \mathbb{D}}\left\|f_{w}\right\|_{F(p, q, s)}<\infty$ and $f_{w} \in F_{0}(p, q, s)$ for every $w \in \mathbb{D}$.

Proof First, $\sup _{w \in \mathbb{D}}\left\|f_{w}\right\|_{F(p, q, s)}<\infty$ was proved in [36]. Second, $f_{w} \in F_{0}(p, q, s)$ for every $w \in \mathbb{D}$ is possibly a known statement too; however, we have not managed to find it in the literature, so we give a proof. Let

$$
I_{f}(a)=\int_{\mathbb{D}}\left|f^{\prime}(z)\right|^{p}\left(1-|z|^{2}\right)^{q}\left(1-\left|\eta_{a}(z)\right|^{2}\right)^{s} d A(z)
$$

If $s>1$, then there is $\varepsilon \in(0, s)$ such that $2+q-\varepsilon>0$. Then by Proposition 1.4.10 in [37] with $n=1$ and some elementary inequalities, since $s+\varepsilon-2>-1$ and $s-\varepsilon>0$, we have that, for $|a|$ close to 1 ,

$$
\begin{aligned}
I_{f_{w}}(a) & =\left(\frac{2+q}{p}\right)^{p}|w|^{p} \int_{\mathbb{D}} \frac{\left(1-|w|^{2}\right)^{p}}{|1-\bar{w} z|^{2+q+p}}\left(1-|z|^{2}\right)^{q}\left(1-\left|\eta_{a}(z)\right|^{2}\right)^{s} d A(z) \\
& \lesssim \int_{\mathbb{D}} \frac{\left(1-|a|^{2}\right)^{s}\left(1-|z|^{2}\right)^{q+s}}{|1-\bar{w} z|^{2+q}|1-\bar{a} z|^{2 s}} d A(z) \\
& \lesssim \frac{\left(1-|a|^{2}\right)^{s}}{(1-|w|)^{\varepsilon}} \int_{\mathbb{D}} \frac{\left(1-|z|^{2}\right)^{s+\varepsilon-2}}{|1-\bar{a} z|^{2 s}} d A(z) \\
& \lesssim \frac{\left(1-|a|^{2}\right)^{\varepsilon}}{(1-|w|)^{\varepsilon}} \rightarrow 0 \quad \text { as }|a| \rightarrow 1^{-}
\end{aligned}
$$

from which the statement follows in this case.

Now assume that $0<s \leq 1$. Let $u$ and $u^{\prime}$ be chosen such that

$$
\max \left\{1, \frac{1}{q+2}\right\}<u<\frac{1}{1-s} \text { and } \quad \frac{1}{u}+\frac{1}{u^{\prime}}=1
$$

and let $v=q+\frac{2}{u^{\prime}}($ for $s=1$, we set $1 /(1-s)=+\infty)$. 
Let $\varepsilon \in(0, s)$ be such that

$$
(v-\varepsilon) u>-1 \quad \text { and } \quad(q+s+\varepsilon-v) u^{\prime}>-1
$$

(it is not difficult to see that such $\varepsilon>0$ exists). Then, using the Hölder inequality, Proposition 1.4.10 in [37] with $n=1,(4)$, and the assumption $s-\varepsilon>0$, we have

$$
\begin{aligned}
I_{f_{w}}(a) \lesssim & \int_{\mathbb{D}} \frac{\left(1-|w|^{2}\right)^{p}\left(1-|a|^{2}\right)^{s}\left(1-|z|^{2}\right)^{q+s}}{|1-\bar{w} z|^{2+q+p}|1-\bar{a} z|^{2 s}} d A(z) \\
= & \int_{\mathbb{D}} \frac{\left(1-|w|^{2}\right)^{p}\left(1-|a|^{2}\right)^{s}\left(1-|z|^{2}\right)^{(q+s+\varepsilon-v)+v-\varepsilon}}{|1-\bar{w} z|^{2+q+p}|1-\bar{a} z|^{2 s}} d A(z) \\
\leq & \left(1-|w|^{2}\right)^{p}\left(\int_{\mathbb{D}} \frac{\left(1-|z|^{2}\right)^{(v-\varepsilon) u}}{|1-\bar{w} z|^{(2+q+p) u}} d A(z)\right)^{1 / u} \\
& \times\left(1-|a|^{2}\right)^{s}\left(\int_{\mathbb{D}} \frac{\left(1-|z|^{2}\right)^{(q+s+\varepsilon-v) u^{\prime}}}{|1-\bar{a} z|^{2 s u^{\prime}}} d A(z)\right)^{1 / u^{\prime}} \\
\lesssim & \frac{\left(1-|a|^{2}\right)^{\varepsilon}}{(1-|w|)^{\varepsilon}} \rightarrow 0, \quad \text { as }|a| \rightarrow 1^{-},
\end{aligned}
$$

from which the statement follows in this case.

Our first result gives some characterizations for the boundedness of the operator $T_{\psi_{1}, \psi_{2}, \varphi}^{n}: F(p, q, s)\left(\right.$ or $\left.F_{0}(p, q, s)\right) \rightarrow \mathcal{B}_{\mu}$.

Theorem 1 Let $0<p, s<\infty,-2<q<\infty, q+s>-1, \psi_{1}, \psi_{2} \in H(\mathbb{D}), n \in \mathbb{N}_{0}, \mu$ be a typical weight, and $\varphi \in S(\mathbb{D})$. Then the following statements are true.

(i) If $n \in \mathbb{N}$, or $n=0$ and $p<2+q$, then $T_{\psi_{1}, \psi_{2}, \varphi}^{n}: F(p, q, s)\left(\right.$ or $\left.F_{0}(p, q, s)\right) \rightarrow \mathcal{B}_{\mu}$ is bounded if and only if

(a) $M_{1}:=\sup _{z \in \mathbb{D}} \frac{\mu(z)\left|\psi_{1}^{\prime}(z)\right|}{\left(1-|\varphi(z)|^{2}\right)^{\frac{2+q}{p}+n-1}}<\infty$,

(b) $M_{2}:=\sup _{z \in \mathbb{D}} \frac{\mu(z)\left|\psi_{1}(z) \varphi^{\prime}(z)+\psi_{2}^{\prime}(z)\right|}{\left(1-|\varphi(z)|^{2}\right)^{\frac{2+q}{p}+n}}<\infty$, and

(c) $M_{3}:=\sup _{z \in \mathbb{D}} \frac{\mu(z)\left|\psi_{2}(z) \varphi^{\prime}(z)\right|}{\left(1-|\varphi(z)|^{2}\right)^{\frac{2+q}{p}+n+1}}<\infty$.

Moreover, the following asymptotic relations hold:

$$
\begin{aligned}
M_{1}+M_{2}+M_{3} & \lesssim\left\|T_{\psi_{1}, \psi_{2}, \varphi}^{n}\right\|_{F(p, q, s)\left(\text { or } F_{0}(p, q, s)\right) \rightarrow \mathcal{B}_{\mu}} \\
& \lesssim M_{1}+M_{2}+M_{3}+\frac{\left|\psi_{1}(0)\right|}{\left(1-|\varphi(0)|^{2}\right)^{\frac{2+q}{p}+n-1}}+\frac{\left|\psi_{2}(0)\right|}{\left(1-|\varphi(0)|^{2}\right)^{\frac{2+q}{p}+n}} .
\end{aligned}
$$

(ii) If $p>2+q$, then $T_{\psi_{1}, \psi_{2}, \varphi}^{0}: F(p, q, s)\left(\right.$ or $\left.F_{0}(p, q, s)\right) \rightarrow \mathcal{B}_{\mu}$ is bounded if and only if $\psi_{1} \in$ $\mathcal{B}_{\mu}$,

(d) $M_{4}:=\sup _{z \in \mathbb{D}} \frac{\mu(z)\left|\psi_{1}(z) \varphi^{\prime}(z)+\psi_{2}^{\prime}(z)\right|}{\left(1-|\varphi(z)|^{2}\right)^{\frac{2+q}{p}}}<\infty$, and

(e) $M_{5}:=\sup _{z \in \mathbb{D}} \frac{\mu(z)\left|\psi_{2}(z) \varphi^{\prime}(z)\right|}{\left(1-|\varphi(z)|^{2}\right)^{\frac{2+q}{p}+1}}<\infty$. 
Moreover, the following asymptotic relations hold:

$$
\begin{aligned}
b_{\mu}\left(\psi_{1}\right)+M_{4}+M_{5} & \lesssim\left\|T_{\psi_{1}, \psi_{2}, \varphi}^{0}\right\|_{F(p, q, s)\left(\text { or } F_{0}(p, q, s)\right) \rightarrow \mathcal{B}_{\mu}} \\
& \lesssim b_{\mu}\left(\psi_{1}\right)+M_{4}+M_{5}+\left|\psi_{1}(0)\right|+\frac{\left|\psi_{2}(0)\right|}{\left(1-|\varphi(0)|^{2}\right)^{\frac{2+q}{p}}} .
\end{aligned}
$$

(iii) If $p=2+q$ and $s>1$, then $T_{\psi_{1}, \psi_{2}, \varphi}^{0}: F(p, q, s)\left(\right.$ or $\left.F_{0}(p, q, s)\right) \rightarrow \mathcal{B}_{\mu}$ is bounded if and only if

(f) $M_{6}:=\sup _{z \in \mathbb{D}} \mu(z)\left|\psi_{1}^{\prime}(z)\right| \ln \left(\frac{2}{1-|\varphi(z)|^{2}}\right)<\infty$,

(g) $M_{7}:=\sup _{z \in \mathbb{D}} \frac{\mu(z)\left|\psi_{1}(z) \varphi^{\prime}(z)+\psi_{2}^{\prime}(z)\right|}{1-|\varphi(z)|^{2}}<\infty$, and

(h) $M_{8}:=\sup _{z \in \mathbb{D}} \frac{\mu(z)\left|\psi_{2}(z) \varphi^{\prime}(z)\right|}{\left(1-|\varphi(z)|^{2}\right)^{2}}<\infty$.

Moreover, the following asymptotic relations hold:

$$
\begin{aligned}
M_{6}+M_{7}+M_{8} & \lesssim\left\|T_{\psi_{1}, \psi_{2}, \varphi}^{0}\right\|_{F(p, q, s)\left(\text { or } F_{0}(p, q, s)\right) \rightarrow \mathcal{B}_{\mu}} \\
& \lesssim M_{6}+M_{7}+M_{8}+\left|\psi_{1}(0)\right| \ln \left(\frac{2}{1-|\varphi(0)|^{2}}\right)+\frac{\left|\psi_{2}(0)\right|}{1-|\varphi(0)|^{2}}
\end{aligned}
$$

Proof (i) Suppose that $n \in \mathbb{N}$, or $n=0$ and $p<2+q$, and that conditions (a), (b), and (c) hold. Then by Lemma 3 , for arbitrary $z \in \mathbb{D}$ and $f \in F(p, q, s)$, we have

$$
\begin{aligned}
\mu(z)\left|\left(T_{\psi_{1}, \psi_{2}, \varphi}^{n} f\right)^{\prime}(z)\right|= & \mu(z) \mid \psi_{1}^{\prime}(z) f^{(n)}(\varphi(z))+\left(\psi_{1}(z) \varphi^{\prime}(z)+\psi_{2}^{\prime}(z)\right) f^{(n+1)}(\varphi(z)) \\
& +\psi_{2}(z) \varphi^{\prime}(z) f^{(n+2)}(\varphi(z)) \mid \\
\lesssim & \mu(z)\left(\frac{\left|\psi_{1}^{\prime}(z)\right|}{\left(1-|\varphi(z)|^{2}\right)^{\frac{2+q}{p}+n-1}}+\frac{\left|\psi_{1}(z) \varphi^{\prime}(z)+\psi_{2}^{\prime}(z)\right|}{\left(1-|\varphi(z)|^{2}\right)^{\frac{2+q}{p}+n}}\right. \\
& \left.+\frac{\left|\psi_{2}(z) \varphi^{\prime}(z)\right|}{\left(1-|\varphi(z)|^{2}\right)^{\frac{2+q}{p}+n+1}}\right)\|f\|_{F(p, q, s) .}
\end{aligned}
$$

On the other hand, we have

$$
\begin{aligned}
\left|\left(T_{\psi_{1}, \psi_{2}, \varphi}^{n} f\right)(0)\right| & =\left|\psi_{1}(0) f^{(n)}(\varphi(0))+\psi_{2}(0) f^{(n+1)}(\varphi(0))\right| \\
& \lesssim\left(\frac{\left|\psi_{1}(0)\right|}{\left(1-|\varphi(0)|^{2}\right)^{\frac{2+q}{p}+n-1}}+\frac{\left|\psi_{2}(0)\right|}{\left(1-|\varphi(0)|^{2}\right)^{\frac{2+q}{p}+n}}\right)\|f\|_{F(p, q, s)} .
\end{aligned}
$$

Hence, from (8) and (9) we obtain that $T_{\psi_{1}, \psi_{2}, \varphi}^{n}: F(p, q, s)\left(\right.$ or $\left.F_{0}(p, q, s)\right) \rightarrow \mathcal{B}_{\mu}$ is bounded and

$$
\begin{aligned}
\left\|T_{\psi_{1}, \psi_{2}, \varphi}^{n}\right\|_{F(p, q, s)\left(\text { or } F_{0}(p, q, s)\right) \rightarrow \mathcal{B}_{\mu}} \lesssim & M_{1}+M_{2}+M_{3} \\
& +\frac{\left|\psi_{1}(0)\right|}{\left(1-|\varphi(0)|^{2}\right)^{\frac{2+q}{p}+n-1}}+\frac{\left|\psi_{2}(0)\right|}{\left(1-|\varphi(0)|^{2}\right)^{\frac{2+q}{p}+n}} .
\end{aligned}
$$

Conversely, suppose that $T_{\psi_{1}, \psi_{2}, \varphi}^{n}: F(p, q, s)\left(\right.$ or $\left.F_{0}(p, q, s)\right) \rightarrow \mathcal{B}_{\mu}$ is bounded. First note that if $T_{\psi_{1}, \psi_{2}, \varphi}^{n}: F(p, q, s) \rightarrow \mathcal{B}_{\mu}$ is bounded, then $T_{\psi_{1}, \psi_{2}, \varphi}^{n}: F_{0}(p, q, s) \rightarrow \mathcal{B}_{\mu}$ is bounded, and

$$
\left\|T_{\psi_{1}, \psi_{2}, \varphi}^{n}\right\|_{F_{0}(p, q, s) \rightarrow \mathcal{B}_{\mu}} \leq\left\|T_{\psi_{1}, \psi_{2}, \varphi}^{n}\right\|_{F(p, q, s) \rightarrow \mathcal{B}_{\mu}} .
$$


Hence, if $T_{\psi_{1}, \psi_{2}, \varphi}^{n}: F_{0}(p, q, s) \rightarrow \mathcal{B}_{\mu}$ is bounded, then we have

$$
\left\|T_{\psi_{1}, \psi_{2}, \varphi}^{n} f\right\|_{\mathcal{B}_{\mu}} \lesssim\left\|T_{\psi_{1}, \psi_{2}, \varphi}^{n}\right\|_{F_{0}(p, q, s) \rightarrow \mathcal{B}_{\mu}}\|f\|_{F(p, q, s)}
$$

for every $f \in F_{0}(p, q, s)$.

Taking $f(z)=z^{n} / n ! \in F_{0}(p, q, s)$ in (12), we obtain that

$$
\sup _{z \in \mathbb{D}} \mu(z)\left|\psi_{1}^{\prime}(z)\right| \lesssim\left\|T_{\psi_{1}, \psi_{2}, \varphi}^{n}\right\|_{F_{0}(p, q, s) \rightarrow \mathcal{B}_{\mu}}
$$

Taking $f(z)=z^{n+1} /(n+1) ! \in F_{0}(p, q, s)$ in (12) and using (13) and the fact that $|\varphi(z)|<1$, we have

$$
\begin{aligned}
\sup _{z \in \mathbb{D}} \mu(z)\left|\psi_{1}(z) \varphi^{\prime}(z)+\psi_{2}^{\prime}(z)\right| & \lesssim\left\|T_{\psi_{1}, \psi_{2}, \varphi}^{n}\right\|_{F_{0}(p, q, s) \rightarrow \mathcal{B}_{\mu}}+\sup _{z \in \mathbb{D}} \mu(z)\left|\psi_{1}^{\prime}(z)\right| \\
& \lesssim\left\|T_{\psi_{1}, \psi_{2}, \varphi}^{n}\right\|_{F_{0}(p, q, s) \rightarrow \mathcal{B}_{\mu}} .
\end{aligned}
$$

Taking $f(z)=z^{n+2} /(n+2) ! \in F_{0}(p, q, s)$ in (12) and using (13), (14), and the fact that $|\varphi(z)|<1$, we easily get

$$
\sup _{z \in \mathbb{D}} \mu(z)\left|\psi_{2}(z) \varphi^{\prime}(z)\right| \lesssim\left\|T_{\psi_{1}, \psi_{2}, \varphi}^{n}\right\|_{F_{0}(p, q, s) \rightarrow \mathcal{B}_{\mu}}
$$

For $\zeta \in \mathbb{D}$ and $n \in \mathbb{N}_{0}$, consider the family of functions

$$
\begin{aligned}
f_{\zeta}(z)= & \prod_{j=1}^{n}\left(\frac{2+q}{p}+j\right)\left(\frac{2+q}{p}+j+1\right) \frac{1-|\varphi(\zeta)|^{2}}{(1-\overline{\varphi(\zeta)} z)^{\frac{2+q}{p}}} \\
& -2 \prod_{j=1}^{n}\left(\frac{2+q}{p}+j-1\right)\left(\frac{2+q}{p}+j+1\right) \frac{\left(1-|\varphi(\zeta)|^{2}\right)^{2}}{(1-\overline{\varphi(\zeta)} z)^{\frac{2+q}{p}+1}} \\
& +\prod_{j=1}^{n}\left(\frac{2+q}{p}+j-1\right)\left(\frac{2+q}{p}+j\right) \frac{\left(1-|\varphi(\zeta)|^{3}\right)^{3}}{(1-\overline{\varphi(\zeta)} z)^{\frac{2+q}{p}+2}}
\end{aligned}
$$

Then by Lemma 4 it is easy to $\operatorname{see}$ that $\sup _{\zeta \in \mathbb{D}}\left\|f_{\zeta}\right\|_{F(p, q, s)} \lesssim 1$ and that $f_{\zeta} \in F_{0}(p, q, s)$ for every $\zeta \in \mathbb{D}$.

We also have

$$
\begin{aligned}
f_{\zeta}^{(n)}(z)= & \prod_{j=1}^{n}\left(\frac{2+q}{p}+j-1\right)\left(\frac{2+q}{p}+j\right)\left(\frac{2+q}{p}+j+1\right)(\overline{\varphi(\zeta)})^{n} \\
& \times\left(\frac{1-|\varphi(\zeta)|^{2}}{(1-\overline{\varphi(\zeta)} z)^{\frac{2+q}{p}+n}}-2 \frac{\left(1-|\varphi(\zeta)|^{2}\right)^{2}}{(1-\overline{\varphi(\zeta)} z)^{\frac{2+q}{p}+n+1}}+\frac{\left(1-|\varphi(\zeta)|^{3}\right)^{3}}{(1-\overline{\varphi(\zeta)} z)^{\frac{2+q}{p}+n+2}}\right) \\
f_{\zeta}^{(n+1)}(z)= & \prod_{j=1}^{n}\left(\frac{2+q}{p}+j-1\right)\left(\frac{2+q}{p}+j\right)\left(\frac{2+q}{p}+j+1\right)\left(\overline{\varphi(\zeta))^{n+1}}\right. \\
& \times\left(\left(\frac{2+q}{p}+n\right) \frac{1-|\varphi(\zeta)|^{2}}{(1-\overline{\varphi(\zeta)} z)^{\frac{2+q}{p}+n+1}}\right.
\end{aligned}
$$




$$
\begin{aligned}
& -2\left(\frac{2+q}{p}+n+1\right) \frac{\left(1-|\varphi(\zeta)|^{2}\right)^{2}}{(1-\overline{\varphi(\zeta)} z)^{\frac{2+q}{p}+n+2}} \\
& \left.+\left(\frac{2+q}{p}+n+2\right) \frac{\left(1-|\varphi(\zeta)|^{3}\right)^{3}}{(1-\overline{\varphi(\zeta)} z)^{\frac{2+q}{p}+n+3}}\right),
\end{aligned}
$$

and

$$
\begin{aligned}
f_{\zeta}^{(n+2)}(z)= & \prod_{j=1}^{n}\left(\frac{2+q}{p}+j-1\right)\left(\frac{2+q}{p}+j\right)\left(\frac{2+q}{p}+j+1\right)(\overline{\varphi(\zeta)})^{n+2} \\
& \times\left(\left(\frac{2+q}{p}+n\right)\left(\frac{2+q}{p}+n+1\right) \frac{1-|\varphi(\zeta)|^{2}}{(1-\overline{\varphi(\zeta)} z)^{\frac{2+q}{p}}+n+2}\right. \\
& -2\left(\frac{2+q}{p}+n+1\right)\left(\frac{2+q}{p}+n+2\right) \frac{\left(1-|\varphi(\zeta)|^{2}\right)^{2}}{(1-\overline{\varphi(\zeta)} z)^{\frac{2+q}{p}+n+3}} \\
& \left.+\left(\frac{2+q}{p}+n+2\right)\left(\frac{2+q}{p}+n+3\right) \frac{\left(1-|\varphi(\zeta)|^{3}\right)^{3}}{(1-\overline{\varphi(\zeta)} z)^{\frac{2+q}{p}}+n+4}\right)
\end{aligned}
$$

Hence,

$$
f_{\zeta}^{(n)}(\varphi(\zeta))=0, \quad f_{\zeta}^{(n+1)}(\varphi(\zeta))=0
$$

and

$$
f_{\zeta}^{(n+2)}(\varphi(\zeta))=\prod_{j=1}^{n}\left(\frac{2+q}{p}+j-1\right)\left(\frac{2+q}{p}+j\right)\left(\frac{2+q}{p}+j+1\right) \frac{2(\overline{\varphi(\zeta)})^{n+2}}{\left(1-|\varphi(\zeta)|^{2}\right)^{\frac{2+q}{p}+n+1}},
$$

which, along with the boundedness of the operator, implies that

$$
\begin{aligned}
\left\|T_{\psi_{1}, \psi_{2}, \varphi}^{n}\right\|_{F_{0}(p, q, s) \rightarrow \mathcal{B}_{\mu}} \gtrsim & \left\|T_{\psi_{1}, \psi_{2}, \varphi}^{n} f_{\zeta}\right\|_{\mathcal{B}_{\mu}} \\
\gtrsim & \mu(\zeta) \mid \psi_{1}^{\prime}(\zeta) f_{\zeta}^{(n)}(\varphi(\zeta))+\left(\psi_{1}(\zeta) \varphi^{\prime}(\zeta)+\psi_{2}^{\prime}(\zeta)\right) f_{\zeta}^{(n+1)}(\varphi(\zeta)) \\
& +\psi_{2}(\zeta) \varphi^{\prime}(\zeta) f_{\zeta}^{(n+2)}(\varphi(\zeta)) \mid \\
= & C_{1}(p, q, n) \mu(\zeta) \frac{\left|\psi_{2}(\zeta) \varphi^{\prime}(\zeta)(\varphi(\zeta))^{n+2}\right|}{\left(1-|\varphi(\zeta)|^{2}\right)^{\frac{2+q}{p}+n+1}}
\end{aligned}
$$

where

$$
C_{1}(p, q, n)=2 \prod_{j=1}^{n}\left(\frac{2+q}{p}+j-1\right)\left(\frac{2+q}{p}+j\right)\left(\frac{2+q}{p}+j+1\right) .
$$

From (15) and (16), for $\delta \in(0,1)$, we obtain

$$
\begin{aligned}
& \sup _{\zeta \in \mathbb{D}} \frac{\mu(\zeta)\left|\psi_{2}(\zeta) \varphi^{\prime}(\zeta)\right|}{\left(1-|\varphi(\zeta)|^{2}\right)^{\frac{2+q}{p}+n+1}} \\
& \quad \leq \sup _{|\varphi(\zeta)|>\delta} \frac{\mu(\zeta)\left|\psi_{2}(\zeta) \varphi^{\prime}(\zeta)\right|}{\left(1-|\varphi(\zeta)|^{2}\right)^{\frac{2+q}{p}+n+1}}+\sup _{|\varphi(\zeta)| \leq \delta} \frac{\mu(\zeta)\left|\psi_{2}(\zeta) \varphi^{\prime}(\zeta)\right|}{\left(1-|\varphi(\zeta)|^{2}\right)^{\frac{2+q}{p}+n+1}}
\end{aligned}
$$




$$
\begin{aligned}
\leq & \frac{1}{\delta^{n+2}} \sup _{|\varphi(\zeta)|>\delta} \frac{\mu(\zeta)\left|\psi_{2}(\zeta) \varphi^{\prime}(\zeta)(\varphi(\zeta))^{n+2}\right|}{\left(1-|\varphi(\zeta)|^{2}\right)^{\frac{2+q}{p}+n+1}} \\
& +\frac{1}{\left(1-\delta^{2}\right)^{\frac{2+q}{p}+n+1}} \sup _{|\varphi(\zeta)| \leq \delta} \mu(\zeta)\left|\psi_{2}(\zeta) \varphi^{\prime}(\zeta)\right| \\
\lesssim & \left(\frac{1}{\delta^{n+2}}+\frac{1}{\left(1-\delta^{2}\right)^{\frac{2+q}{p}+n+1}}\right)\left\|T_{\psi_{1}, \psi_{2}, \varphi}^{n}\right\|_{F_{0}(p, q, s) \rightarrow \mathcal{B}_{\mu}} .
\end{aligned}
$$

From this it follows that (c) holds and, moreover,

$$
M_{3} \lesssim\left\|T_{\psi_{1}, \psi_{2}, \varphi}^{n}\right\|_{F_{0}(p, q, s) \rightarrow \mathcal{B}_{\mu}}
$$

For $\zeta \in \mathbb{D}$ and $n \in \mathbb{N}_{0}$, consider the family of functions

$$
\begin{aligned}
g_{\zeta}(z)= & \left(\frac{2+q}{p}+n+2\right) \prod_{j=1}^{n}\left(\frac{2+q}{p}+j\right)\left(\frac{2+q}{p}+j+1\right) \frac{1-|\varphi(\zeta)|^{2}}{(1-\overline{\varphi(\zeta)} z)^{\frac{2+q}{p}}} \\
& -\left(2 \frac{(2+q)}{p}+2 n+3\right) \prod_{j=1}^{n}\left(\frac{2+q}{p}+j-1\right)\left(\frac{2+q}{p}+j+1\right) \frac{\left(1-|\varphi(\zeta)|^{2}\right)^{2}}{(1-\overline{\varphi(\zeta)} z)^{\frac{2+q}{p}+1}} \\
& +\left(\frac{2+q}{p}+n+1\right) \prod_{j=1}^{n}\left(\frac{2+q}{p}+j-1\right)\left(\frac{2+q}{p}+j\right) \frac{\left(1-|\varphi(\zeta)|^{3}\right)^{3}}{(1-\overline{\varphi(\zeta)} z)^{\frac{2+q}{p}+2}} .
\end{aligned}
$$

Then by Lemma 4 it is easy to see that $\sup _{\zeta \in \mathbb{D}}\left\|g_{\zeta}\right\|_{F(p, q, s)} \lesssim 1$ and that $g_{\zeta} \in F_{0}(p, q, s)$ for every $\zeta \in \mathbb{D}$.

We also have

$$
\begin{aligned}
& g_{\zeta}^{(n)}(z)=\prod_{j=1}^{n}\left(\frac{2+q}{p}+j-1\right)\left(\frac{2+q}{p}+j\right)\left(\frac{2+q}{p}+j+1\right)(\overline{\varphi(\zeta)})^{n} \\
& \times\left(\left(\frac{2+q}{p}+n+2\right) \frac{1-|\varphi(\zeta)|^{2}}{(1-\overline{\varphi(\zeta)} z)^{\frac{2+q}{p}+n}}\right. \\
& -\left(2 \frac{(2+q)}{p}+2 n+3\right) \frac{\left(1-|\varphi(\zeta)|^{2}\right)^{2}}{(1-\overline{\varphi(\zeta)} z)^{\frac{2+q}{p}+n+1}} \\
& \left.+\left(\frac{2+q}{p}+n+1\right) \frac{\left(1-|\varphi(\zeta)|^{3}\right)^{3}}{(1-\overline{\varphi(\zeta) z})^{\frac{2+q}{p}+n+2}}\right), \\
& g_{\zeta}^{(n+1)}(z)=\prod_{j=1}^{n}\left(\frac{2+q}{p}+j-1\right)\left(\frac{2+q}{p}+j\right)\left(\frac{2+q}{p}+j+1\right)(\overline{\varphi(\zeta)})^{n+1} \\
& \times\left(\left(\frac{2+q}{p}+n\right)\left(\frac{2+q}{p}+n+2\right) \frac{1-|\varphi(\zeta)|^{2}}{(1-\overline{\varphi(\zeta)} z)^{\frac{2+q}{p}+n+1}}\right. \\
& -\left(\frac{2+q}{p}+n+1\right)\left(2 \frac{(2+q)}{p}+2 n+3\right) \frac{\left(1-|\varphi(\zeta)|^{2}\right)^{2}}{(1-\overline{\varphi(\zeta)} z)^{\frac{2+q}{p}+n+2}} \\
& \left.+\left(\frac{2+q}{p}+n+2\right)\left(\frac{2+q}{p}+n+1\right) \frac{\left(1-|\varphi(\zeta)|^{3}\right)^{3}}{\left(1-\overline{\varphi(\zeta) z)^{\frac{2+q}{p}}+n+3}\right.}\right),
\end{aligned}
$$


and

$$
\begin{aligned}
g_{\zeta}^{(n+2)}(z)= & \prod_{j=1}^{n}\left(\frac{2+q}{p}+j-1\right)\left(\frac{2+q}{p}+j\right)\left(\frac{2+q}{p}+j+1\right)(\overline{\varphi(\zeta)})^{n+2} \\
& \times\left(\frac{2+q}{p}+n+1\right)\left(\frac{2+q}{p}+n+2\right)\left(\left(\frac{2+q}{p}+n\right) \frac{1-|\varphi(\zeta)|^{2}}{(1-\overline{\varphi(\zeta)} z)^{\frac{2+q}{p}+n+2}}\right. \\
& -\left(2 \frac{(2+q)}{p}+2 n+3\right) \frac{\left(1-|\varphi(\zeta)|^{2}\right)^{2}}{(1-\overline{\varphi(\zeta)} z)^{\frac{2+q}{p}+n+3}} \\
& \left.+\left(\frac{2+q}{p}+n+3\right) \frac{\left(1-|\varphi(\zeta)|^{3}\right)^{3}}{(1-\overline{\varphi(\zeta)} z)^{\frac{2+q}{p}}+n+4}\right) .
\end{aligned}
$$

Hence,

$$
g_{\zeta}^{(n)}(\varphi(\zeta))=0, \quad g_{\zeta}^{(n+2)}(\varphi(\zeta))=0,
$$

and

$$
g_{\zeta}^{(n+1)}(\varphi(\zeta))=-\prod_{j=1}^{n}\left(\frac{2+q}{p}+j-1\right)\left(\frac{2+q}{p}+j\right)\left(\frac{2+q}{p}+j+1\right) \frac{(\overline{\varphi(\zeta)})^{n+1}}{\left(1-|\varphi(\zeta)|^{2}\right)^{\frac{2+q}{p}+n}},
$$

which, along with the boundedness of the operator $T_{\psi_{1}, \psi_{2}, \varphi}^{n}: F_{0}(p, q, s) \rightarrow \mathcal{B}_{\mu}$, implies that

$$
\begin{aligned}
\| & T_{\psi_{1}, \psi_{2}, \varphi}^{n} \|_{F_{0}(p, q, s) \rightarrow \mathcal{B}_{\mu}} \\
& \gtrsim\left\|T_{\psi_{1}, \psi_{2}, \varphi}^{n} g_{\zeta}\right\|_{\mathcal{B}_{\mu}} \\
& \gtrsim \mu(\zeta) \mid \psi_{1}^{\prime}(\zeta) g_{\zeta}^{(n)}(\varphi(\zeta))+\left(\psi_{1}(\zeta) \varphi^{\prime}(\zeta)+\psi_{2}^{\prime}(\zeta)\right) g^{(n+1)}(\varphi(\zeta)) \\
& \quad+\psi_{2}(\zeta) \varphi^{\prime}(\zeta) g^{(n+2)}(\varphi(\zeta)) \mid \\
= & C_{2}(p, q, n) \mu(\zeta) \frac{\left|\psi_{1}(\zeta) \varphi^{\prime}(\zeta)+\psi_{2}^{\prime}(\zeta) \| \varphi(\zeta)\right|^{n+1}}{\left(1-|\varphi(\zeta)|^{2}\right)^{\frac{2+q}{p}+n}},
\end{aligned}
$$

where

$$
C_{2}(p, q, n)=\prod_{j=1}^{n}\left(\frac{2+q}{p}+j-1\right)\left(\frac{2+q}{p}+j\right)\left(\frac{2+q}{p}+j+1\right) .
$$

From (14) and (18), for $\delta \in(0,1)$, we obtain

$$
\begin{aligned}
& \sup _{\zeta \in \mathbb{D}} \frac{\mu(\zeta)\left|\psi_{1}(\zeta) \varphi^{\prime}(\zeta)+\psi_{2}^{\prime}(\zeta)\right|}{\left(1-|\varphi(\zeta)|^{2}\right)^{\frac{2+q}{p}+n}} \\
& \quad \leq \sup _{|\varphi(\zeta)|>\delta} \frac{\mu(\zeta)\left|\psi_{1}(\zeta) \varphi^{\prime}(\zeta)+\psi_{2}^{\prime}(\zeta)\right|}{\left(1-|\varphi(\zeta)|^{2}\right)^{\frac{2+q}{p}}+n}+\sup _{|\varphi(\zeta)| \leq \delta} \frac{\mu(\zeta)\left|\psi_{1}(\zeta) \varphi^{\prime}(\zeta)+\psi_{2}^{\prime}(\zeta)\right|}{\left(1-|\varphi(\zeta)|^{2}\right)^{\frac{2+q}{p}+n}} \\
& \quad \leq \frac{1}{\delta^{n+1}} \sup _{|\varphi(\zeta)|>\delta} \frac{\mu(\zeta)\left|\psi_{1}(\zeta) \varphi^{\prime}(\zeta)+\psi_{2}^{\prime}(\zeta)\right||\varphi(\zeta)|^{n+1}}{\left(1-|\varphi(\zeta)|^{2}\right)^{\frac{2+q}{p}+n}}
\end{aligned}
$$




$$
\begin{aligned}
& +\frac{1}{\left(1-\delta^{2}\right)^{\frac{2+q}{p}+n}} \sup _{|\varphi(\zeta)| \leq \delta} \mu(\zeta)\left|\psi_{1}(\zeta) \varphi^{\prime}(\zeta)+\psi_{2}^{\prime}(\zeta)\right| \\
\lesssim & \left(\frac{1}{\delta^{n+1}}+\frac{1}{\left(1-\delta^{2}\right)^{\frac{2+q}{p}+n}}\right)\left\|T_{\psi_{1}, \psi_{2}, \varphi}^{n}\right\|_{F_{0}(p, q, s) \rightarrow \mathcal{B}_{\mu}} .
\end{aligned}
$$

From this it follows that (b) holds and, moreover,

$$
M_{2} \lesssim\left\|T_{\psi_{1}, \psi_{2}, \varphi}^{n}\right\|_{F_{0}(p, q, s) \rightarrow \mathcal{B}_{\mu}}
$$

Finally, for $\zeta \in \mathbb{D}$ and $n \in \mathbb{N}_{0}$, consider the family of functions

$$
\begin{aligned}
h_{\zeta}(z)= & \left(\frac{2+q}{p}+n+1\right)\left(\frac{2+q}{p}+n+2\right) \\
& \times \prod_{j=1}^{n}\left(\frac{2+q}{p}+j\right)\left(\frac{2+q}{p}+j+1\right) \frac{1-|\varphi(\zeta)|^{2}}{(1-\overline{\varphi(\zeta)} z)^{\frac{2+q}{p}}} \\
& -2\left(\frac{2+q}{p}+n\right)\left(\frac{2+q}{p}+n+2\right) \\
& \times \prod_{j=1}^{n}\left(\frac{2+q}{p}+j-1\right)\left(\frac{2+q}{p}+j+1\right) \frac{\left(1-|\varphi(\zeta)|^{2}\right)^{2}}{(1-\overline{\varphi(\zeta)} z)^{\frac{2+q}{p}+1}} \\
& +\left(\frac{2+q}{p}+n\right)\left(\frac{2+q}{p}+n+1\right) \\
& \times \prod_{j=1}^{n}\left(\frac{2+q}{p}+j-1\right)\left(\frac{2+q}{p}+j\right) \frac{\left(1-|\varphi(\zeta)|^{3}\right)^{3}}{(1-\overline{\varphi(\zeta)} z)^{\frac{2+q}{p}+2}} .
\end{aligned}
$$

Then, by Lemma 4 it is easy to see that $\sup _{\zeta \in \mathbb{D}}\left\|h_{\zeta}\right\|_{F(p, q, s)} \lesssim 1$ and that $h_{\zeta} \in F_{0}(p, q, s)$ for every $\zeta \in \mathbb{D}$. We also have

$$
\begin{aligned}
h_{\zeta}^{(n)}(z)= & \prod_{j=1}^{n}\left(\frac{2+q}{p}+j-1\right)\left(\frac{2+q}{p}+j\right)\left(\frac{2+q}{p}+j+1\right)\left(\overline{\varphi(\zeta))^{n}}\right. \\
& \times\left(\left(\frac{2+q}{p}+n+1\right)\left(\frac{2+q}{p}+n+2\right) \frac{1-|\varphi(\zeta)|^{2}}{(1-\overline{\varphi(\zeta)} z)^{\frac{2+q}{p}+n}}\right. \\
& -2\left(\frac{2+q}{p}+n\right)\left(\frac{2+q}{p}+n+2\right) \frac{\left(1-|\varphi(\zeta)|^{2}\right)^{2}}{(1-\overline{\varphi(\zeta)} z)^{\frac{2+q}{p}+n+1}} \\
& \left.+\left(\frac{2+q}{p}+n\right)\left(\frac{2+q}{p}+n+1\right) \frac{\left(1-|\varphi(\zeta)|^{3}\right)^{3}}{(1-\overline{\varphi(\zeta)} z)^{\frac{2+q}{p}}+n+2}\right), \\
h_{\zeta}^{(n+1)}(z)= & \prod_{j=1}^{n}\left(\frac{2+q}{p}+j-1\right)\left(\frac{2+q}{p}+j\right)\left(\frac{2+q}{p}+j+1\right)\left(\overline{\varphi(\zeta))^{n+1}}\right. \\
& \times\left(\frac{2+q}{p}+n\right)\left(\frac{2+q}{p}+n+1\right)\left(\frac{2+q}{p}+n+2\right) \\
& \times\left(\frac{1-|\varphi(\zeta)|^{2}}{(1-\overline{\varphi(\zeta)} z)^{\frac{2+q}{p}+n+1}}-\frac{2\left(1-|\varphi(\zeta)|^{2}\right)^{2}}{(1-\overline{\varphi(\zeta)} z)^{\frac{2+q}{p}+n+2}}+\frac{\left(1-|\varphi(\zeta)|^{3}\right)^{3}}{(1-\overline{\varphi(\zeta)} z)^{\frac{2+q}{p}+n+3}}\right),
\end{aligned}
$$


and

$$
\begin{aligned}
h_{\zeta}^{(n+2)}(z)= & \prod_{j=1}^{n}\left(\frac{2+q}{p}+j-1\right)\left(\frac{2+q}{p}+j\right)\left(\frac{2+q}{p}+j+1\right)(\overline{\varphi(\zeta)})^{n+2} \\
& \times\left(\frac{2+q}{p}+n\right)\left(\frac{2+q}{p}+n+1\right)\left(\frac{2+q}{p}+n+2\right) \\
& \times\left(\left(\frac{2+q}{p}+n+1\right) \frac{1-|\varphi(\zeta)|^{2}}{(1-\overline{\varphi(\zeta)} z)^{\frac{2+q}{p}+n+2}}\right. \\
& -2\left(\frac{2+q}{p}+n+2\right) \frac{\left(1-|\varphi(\zeta)|^{2}\right)^{2}}{(1-\overline{\varphi(\zeta)} z)^{\frac{2+q}{p}+n+3}} \\
& \left.+\left(\frac{2+q}{p}+n+3\right) \frac{\left(1-|\varphi(\zeta)|^{3}\right)^{3}}{\left(1-\overline{\varphi(\zeta) z)^{\frac{2+q}{p}}+n+4}\right.}\right) .
\end{aligned}
$$

Hence,

$$
h_{\zeta}^{(n+1)}(\varphi(\zeta))=0, \quad h_{\zeta}^{(n+2)}(\varphi(\zeta))=0,
$$

and

$$
h_{\zeta}^{(n)}(\varphi(\zeta))=C_{3}(p, q, n) \frac{(\overline{\varphi(\zeta)})^{n}}{\left(1-|\varphi(\zeta)|^{2}\right)^{\frac{2+q}{p}+n-1}},
$$

where

$$
C_{3}(p, q, n)=2 \prod_{j=1}^{n}\left(\frac{2+q}{p}+j-1\right)\left(\frac{2+q}{p}+j\right)\left(\frac{2+q}{p}+j+1\right) .
$$

Thus, using the boundedness of the operator, we have

$$
\begin{aligned}
\left\|T_{\psi_{1}, \psi_{2}, \varphi}^{n}\right\|_{F_{0}(p, q, s) \rightarrow \mathcal{B}_{\mu}} \gtrsim & \left\|T_{\psi_{1}, \psi_{2}, \varphi}^{n} h_{\zeta}\right\|_{\mathcal{B}_{\mu}} \\
\gtrsim & \mu(\zeta) \mid \psi_{1}^{\prime}(\zeta) h_{\zeta}^{(n)}(\varphi(\zeta))+\left(\psi_{1}(\zeta) \varphi^{\prime}(\zeta)+\psi_{2}^{\prime}(\zeta)\right) h_{\zeta}^{(n+1)}(\varphi(\zeta)) \\
& +\psi_{2}(\zeta) \varphi^{\prime}(\zeta) h_{\zeta}^{(n+2)}(\varphi(\zeta)) \mid \\
\geq & C_{3}(p, q, n) \mu(\zeta) \frac{\left|\psi_{1}^{\prime}(\zeta)(\varphi(\zeta))^{n}\right|}{\left(1-|\varphi(\zeta)|^{2}\right)^{\frac{2+q}{p}+n-1}} .
\end{aligned}
$$

From (13) and (20), for $\delta \in(0,1)$, we obtain

$$
\begin{aligned}
& \sup _{\zeta \in \mathbb{D}} \frac{\mu(\zeta)\left|\psi_{1}^{\prime}(\zeta)\right|}{\left(1-|\varphi(\zeta)|^{2}\right)^{\frac{2+q}{p}+n-1}} \\
& \quad \leq \sup _{|\varphi(\zeta)|>\delta} \frac{\mu(\zeta)\left|\psi_{1}^{\prime}(\zeta)\right|}{\left(1-|\varphi(\zeta)|^{2}\right)^{\frac{2+q}{p}+n-1}}+\sup _{|\varphi(\zeta)| \leq \delta} \frac{\mu(\zeta)\left|\psi_{1}^{\prime}(\zeta)\right|}{\left(1-|\varphi(\zeta)|^{2}\right)^{\frac{2+q}{p}+n-1}} \\
& \quad \leq \frac{1}{\delta^{n}} \sup _{|\varphi(\zeta)|>\delta} \frac{\mu(\zeta)\left|\psi_{1}^{\prime}(\zeta)(\varphi(\zeta))^{n}\right|}{\left(1-|\varphi(\zeta)|^{2}\right)^{\frac{2+q}{p}+n-1}}+\frac{1}{\left(1-\delta^{2}\right)^{\frac{2+q}{p}+n-1}} \sup _{|\varphi(\zeta)| \leq \delta} \mu(\zeta)\left|\psi_{1}^{\prime}(\zeta)\right| \\
& \quad \leq\left(\frac{1}{\delta^{n}}+\frac{1}{\left(1-\delta^{2}\right)^{\frac{2+q}{p}+n-1}}\right)\left\|T_{\psi_{1}, \psi_{2}, \varphi}^{n}\right\|_{F_{0}(p, q, s) \rightarrow \mathcal{B}_{\mu}} .
\end{aligned}
$$


From this it follows that (a) holds and, moreover,

$$
M_{1} \lesssim\left\|T_{\psi_{1}, \psi_{2}, \varphi}^{n}\right\|_{F_{0}(p, q, s) \rightarrow \mathcal{B}_{\mu}} \cdot
$$

From (17), (19), and (21) we have

$$
M_{1}+M_{2}+M_{3} \lesssim\left\|T_{\psi_{1}, \psi_{2}, \varphi}^{n}\right\|_{F_{0}(p, q, s) \rightarrow \mathcal{B}_{\mu}}
$$

Hence, from (10), (11), and (22) we have that (5) holds, as desired.

(ii) Assume that $n=0, p>2+q, \psi_{1} \in \mathcal{B}_{\mu}$, and that conditions (d) and (e) hold. If $f \in$ $F(p, q, s)$, then by Lemma 3 we have

$$
\begin{aligned}
\mu(z)\left|\left(T_{\psi_{1}, \psi_{2}, \varphi}^{0} f\right)^{\prime}(z)\right| \leq & \mu(z)\left|\psi_{1}^{\prime}(z)\right||f(\varphi(z))|+\mu(z)\left|\psi_{1}(z) \varphi^{\prime}(z)+\psi_{2}^{\prime}(z)\right|\left|f^{\prime}(\varphi(z))\right| \\
& +\mu(z)\left|\psi_{2}(z) \varphi^{\prime}(z)\right|\left|f^{\prime \prime}(\varphi(z))\right| \\
\lesssim & \left(\mu(z)\left|\psi_{1}^{\prime}(z)\right|+\frac{\mu(z)\left|\psi_{1}(z) \varphi^{\prime}(z)+\psi_{2}^{\prime}(z)\right|}{\left(1-|\varphi(z)|^{2}\right)^{\frac{2+q}{p}}}\right. \\
& +\frac{\mu(z)\left|\psi_{2}(z) \varphi^{\prime}(z)\right|}{\left.\left(1-|\varphi(z)|^{2}\right)^{\frac{2+q}{p}+1}\right)\|f\|_{F(p, q, s)}} \\
\lesssim & \left(b_{\mu}\left(\psi_{1}\right)+M_{4}+M_{5}\right)\|f\|_{F(p, q, s)}
\end{aligned}
$$

and

$$
\begin{aligned}
\left|\left(T_{\psi_{1}, \psi_{2}, \varphi}^{0} f\right)(0)\right| & =\left|\psi_{1}(0) f(\varphi(0))+\psi_{2}(0) f^{\prime}(\varphi(0))\right| \\
& \lesssim\left(\left|\psi_{1}(0)\right|+\frac{\left|\psi_{2}(0)\right|}{\left(1-|\varphi(0)|^{2}\right)^{\frac{2+q}{p}}}\right)\|f\|_{F(p, q, s)} .
\end{aligned}
$$

From (23) and (24) we see that $T_{\psi_{1}, \psi_{2}, \varphi}^{0}: F(p, q, s)\left(\right.$ or $\left.F_{0}(p, q, s)\right) \rightarrow \mathcal{B}_{\mu}$ is bounded and that

$$
\left\|T_{\psi_{1}, \psi_{2}, \varphi}^{0}\right\|_{F(p, q, s)\left(\text { or } F_{0}(p, q, s)\right) \rightarrow \mathcal{B}_{\mu}} \lesssim b_{\mu}\left(\psi_{1}\right)+M_{4}+M_{5}+\left|\psi_{1}(0)\right|+\frac{\left|\psi_{2}(0)\right|}{\left(1-|\varphi(0)|^{2}\right)^{\frac{2+q}{p}}}
$$

Conversely, if $T_{\psi_{1}, \psi_{2}, \varphi}^{0}: F(p, q, s) \rightarrow \mathcal{B}_{\mu}$ is bounded, then $T_{\psi_{1}, \psi_{2}, \varphi}^{0}: F_{0}(p, q, s) \rightarrow \mathcal{B}_{\mu}$ is bounded, (11) and (12) hold, and by using the function $f(z) \equiv 1 \in F_{0}(p, q, s)$ therein we obtain that $\psi_{1} \in \mathcal{B}_{\mu}$ and that (13) holds. From the proof of (i) for $n=0$, we see that (d) and (e) hold, as well as the asymptotic relations $M_{4} \lesssim\left\|T_{\psi_{1}, \psi_{2}, \varphi}^{0}\right\|_{F_{0}(p, q, s) \rightarrow \mathcal{B}_{\mu}}$ and $M_{5} \lesssim$ $\left\|T_{\psi_{1}, \psi_{2}, \varphi}^{0}\right\|_{F_{0}(p, q, s) \rightarrow \mathcal{B}_{\mu}}$. Hence,

$$
b_{\mu}\left(\psi_{1}\right)+M_{4}+M_{5} \lesssim\left\|T_{\psi_{1}, \psi_{2}, \varphi}^{0}\right\|_{F_{0}(p, q, s) \rightarrow \mathcal{B}_{\mu}}
$$

from which, along with (11) and (25), we see that (6) holds.

(iii) Assume that $n=0, p=2+q, s>1$, and that conditions (f), (g), and (h) hold. Assume that $f \in F(p, q, s)$. Then by Lemma 3 we have

$$
\begin{aligned}
\mu(z)\left|\left(T_{\psi_{1}, \psi_{2}, \varphi}^{0} f\right)^{\prime}(z)\right| \leq & \mu(z)\left|\psi_{1}^{\prime}(z)\right||f(\varphi(z))|+\mu(z)\left|\psi_{1}(z) \varphi^{\prime}(z)+\psi_{2}(z)\right|\left|f^{\prime}(\varphi(z))\right| \\
& +\mu(z)\left|\psi_{2}(z) \varphi^{\prime}(z)\right|\left|f^{\prime \prime}(\varphi(z))\right|
\end{aligned}
$$




$$
\begin{aligned}
\lesssim & \left(\mu(z)\left|\psi_{1}^{\prime}(z)\right| \ln \left(\frac{2}{1-|\varphi(z)|^{2}}\right)+\frac{\mu(z)\left|\psi_{1}(z) \varphi^{\prime}(z)+\psi_{2}^{\prime}(z)\right|}{1-|\varphi(z)|^{2}}\right. \\
& \left.+\frac{\mu(z)\left|\varphi^{\prime}(z) \psi_{2}(z)\right|}{\left(1-|\varphi(z)|^{2}\right)^{2}}\right)\|f\|_{F(p, q, s)} \\
\lesssim & \left(M_{6}+M_{7}+M_{8}\right)\|f\|_{F(p, q, s)}
\end{aligned}
$$

and

$$
\begin{aligned}
\left|\left(T_{\psi_{1}, \psi_{2}, \varphi}^{0} f\right)(0)\right| & =\left|\psi_{1}(0) f(\varphi(0))+\psi_{2}(0) f^{\prime}(\varphi(0))\right| \\
& \lesssim\left(\left|\psi_{1}(0)\right| \ln \left(\frac{2}{1-|\varphi(0)|^{2}}\right)+\frac{\left|\psi_{2}(0)\right|}{1-|\varphi(0)|^{2}}\right)\|f\|_{F(p, q, s)} .
\end{aligned}
$$

From (26) and (27) we see that $T_{\psi_{1}, \psi_{2}, \varphi}^{0}: F(p, q, s)\left(\right.$ or $\left.F_{0}(p, q, s)\right) \rightarrow \mathcal{B}_{\mu}$ is bounded and that

$$
\left\|T_{\psi_{1}, \psi_{2}, \varphi}^{0}\right\|_{F(p, q, s) \rightarrow \mathcal{B}_{\mu}} \lesssim M_{6}+M_{7}+M_{8}+\left|\psi_{1}(0)\right| \ln \left(\frac{2}{1-|\varphi(0)|^{2}}\right)+\frac{\left|\psi_{2}(0)\right|}{1-|\varphi(0)|^{2}}
$$

Conversely, suppose that $T_{\psi_{1}, \psi_{2}, \varphi}^{0}: F(p, q, s) \rightarrow \mathcal{B}_{\mu}$ is bounded. Then $T_{\psi_{1}, \psi_{2}, \varphi}^{0}: F_{0}(p$, $q, s) \rightarrow \mathcal{B}_{\mu}$ is bounded too. For $\zeta \in \mathbb{D}$, let

$$
f_{\zeta}(z)=\ln \left(\frac{2}{1-\overline{\varphi(\zeta)} z}\right)
$$

Then

$$
f_{\zeta}^{\prime}(z)=\frac{\overline{\varphi(\zeta)}}{1-\overline{\varphi(\zeta)} z} \quad \text { and } \quad f_{\zeta}^{\prime \prime}(z)=\frac{(\overline{\varphi(\zeta)})^{2}}{(1-\overline{\varphi(\zeta)} z)^{2}}
$$

Easy calculation shows that $f_{\zeta} \in \mathcal{B}_{0}^{1}, \zeta \in \mathbb{D}$, and that there is $C>0$ such that $\sup _{\zeta \in \mathbb{D}}\left\|f_{\zeta}\right\|_{\mathcal{B}} \leq$ $C$. Since $s>1$, we have $f_{\zeta} \in F_{0}(p, q, s)$ and $\sup _{\zeta \in \mathbb{D}}\left\|f_{\zeta}\right\|_{F(p, q, s)} \leq C$.

Therefore,

$$
\begin{aligned}
\left\|T_{\psi_{1}, \psi_{2}, \varphi}^{0}\right\|_{F_{0}(p, q, s) \rightarrow \mathcal{B}_{\mu}} \gtrsim & \left\|T_{\psi_{1}, \psi_{2}, \varphi}^{0} f_{\zeta}\right\|_{\mathcal{B}_{\mu}} \geq \mu(\zeta)\left|\left(T_{\psi_{1}, \psi_{2}, \varphi}^{0} f_{\zeta}\right)^{\prime}(\zeta)\right| \\
\geq & \mu(\zeta) \mid \psi_{1}^{\prime}(\zeta) f(\varphi(\zeta))+\left(\psi_{1}(\zeta) \varphi^{\prime}(\zeta)+\psi_{2}^{\prime}(\zeta)\right) f^{\prime}(\varphi(\zeta)) \\
& +\psi_{2}(\zeta) \varphi^{\prime}(\zeta) f^{\prime \prime}(\varphi(\zeta)) \mid \\
= & \mu(\zeta) \mid \psi_{1}^{\prime}(\zeta) \ln \left(\frac{2}{1-|\varphi(\zeta)|^{2}}\right)+\psi_{2}(\zeta) \varphi^{\prime}(\zeta) \frac{(\overline{\varphi(\zeta)})^{2}}{\left(1-|\varphi(\zeta)|^{2}\right)^{2}} \\
& +\left(\psi_{1}(\zeta) \varphi^{\prime}(\zeta)+\psi_{2}^{\prime}(\zeta)\right) \frac{\overline{\varphi(\zeta)}}{1-|\varphi(\zeta)|^{2}} \mid
\end{aligned}
$$

for every $\zeta \in \mathbb{D}$, from which, along with the fact that $|\varphi(\zeta)|<1, \zeta \in \mathbb{D}$, we get

$$
\begin{aligned}
& \mu(\zeta)\left|\psi_{1}^{\prime}(\zeta)\right| \ln \left(\frac{2}{1-|\varphi(\zeta)|^{2}}\right) \\
& \quad \lesssim\left\|T_{\psi_{1}, \psi_{2}, \varphi}^{0}\right\|_{F_{0}(p, q, s) \rightarrow \mathcal{B}_{\mu}}+\frac{\left|\psi_{1}(\zeta) \varphi^{\prime}(\zeta)+\psi_{2}^{\prime}(\zeta)\right|}{1-|\varphi(\zeta)|^{2}}+\frac{\left|\psi_{2}(\zeta) \varphi^{\prime}(\zeta)\right|}{\left(1-|\varphi(\zeta)|^{2}\right)^{2}}
\end{aligned}
$$


From the proof of (i) we see that $(\mathrm{g})$ and $(\mathrm{h})$ hold and that

$$
M_{7} \lesssim\left\|T_{\psi_{1}, \psi_{2}, \varphi}^{0}\right\|_{F_{0}(p, q, s) \rightarrow \mathcal{B}_{\mu}} \quad \text { and } \quad M_{8} \lesssim\left\|T_{\psi_{1}, \psi_{2}, \varphi}^{0}\right\|_{F_{0}(p, q, s) \rightarrow \mathcal{B}_{\mu}} .
$$

Taking the supremum over $\mathbb{D}$ in (30) and then using the last asymptotic relations, we see that

$$
M_{6} \lesssim\left\|T_{\psi_{1}, \psi_{2}, \varphi}^{0}\right\|_{F_{0}(p, q, s) \rightarrow \mathcal{B}_{\mu}} \cdot
$$

Hence, we have $M_{6}+M_{7}+M_{8} \lesssim\left\|T_{\psi_{1}, \psi_{2}, \varphi}^{0}\right\|_{F_{0}(p, q, s) \rightarrow \mathcal{B}_{\mu}}$, which, along with (11) and (28), yields (7), completing the proof of the theorem.

The next lemma is proved by using standard Schwartz's arguments in [38].

Lemma 5 Let $0<p, s<\infty,-2<q<\infty, q+s>-1, \psi_{1}, \psi_{2} \in H(\mathbb{D}), n \in \mathbb{N}_{0}, \mu$ be a typical weight, and $\varphi \in S(\mathbb{D})$. Then $T_{\psi_{1}, \psi_{2}, \varphi}^{n}: F(p, q, s)\left(\right.$ or $\left.F_{0}(p, q, s)\right) \rightarrow \mathcal{B}_{\mu}$ is compact if and only if for every bounded sequence $\left(f_{k}\right)_{k \in \mathbb{N}}$ in $F(p, q, s)\left(\right.$ or $\left.F_{0}(p, q, s)\right)$ that converges to zero on compact subsets of $\mathbb{D}$ as $k \rightarrow \infty$, we have $\left\|T_{\psi_{1}, \psi_{2}, \varphi}^{n} f_{k}\right\|_{\mathcal{B}_{\mu}} \rightarrow 0$ as $k \rightarrow \infty$.

Theorem 2 Let $0<p, s<\infty,-2<q<\infty, q+s>-1, \psi_{1}, \psi_{2} \in H(\mathbb{D}), n \in \mathbb{N}_{0}, \mu$ be a typical weight, and $\varphi \in S(\mathbb{D})$. Then the following statements are true.

(i) If $n \in \mathbb{N}$, or $n=0$ and $p<2+q$, then $T_{\psi_{1}, \psi_{2}, \varphi}^{n}: F(p, q, s)\left(\right.$ or $\left.F_{0}(p, q, s)\right) \rightarrow \mathcal{B}_{\mu}$ is compact if and only if $\psi_{1} \in \mathcal{B}_{\mu}, \psi_{1} \varphi^{\prime}+\psi_{2}^{\prime} \in H_{\mu}^{\infty}, \psi_{2} \varphi^{\prime} \in H_{\mu}^{\infty}$, and

(a) $\lim _{|\varphi(z)| \rightarrow 1} \frac{\mu(z)\left|\psi_{1}^{\prime}(z)\right|}{\left(1-|\varphi(z)|^{2}\right)^{\frac{2+q}{p}}+n-1}=0$,

(b) $\lim _{|\varphi(z)| \rightarrow 1} \frac{\mu(z)\left|\psi_{1}(z) \varphi^{\prime}(z)+\psi_{2}^{\prime}(z)\right|}{\left(1-|\varphi(z)|^{2}\right)^{\frac{2+q}{p}+n}}=0$, and

(c) $\lim _{|\varphi(z)| \rightarrow 1} \frac{\mu(z)\left|\psi_{2}(z) \varphi^{\prime}(z)\right|}{\left(1-|\varphi(z)|^{2}\right)^{\frac{2+}{p}+n-1}}=0$.

(ii) If $p>2+q$, then $T_{\psi_{1}, \psi_{2}, \varphi}^{0}: F(p, q, s)\left(\right.$ or $\left.F_{0}(p, q, s)\right) \rightarrow \mathcal{B}_{\mu}$ is compact if and only if $\psi_{1} \in$ $\mathcal{B}_{\mu}, \psi_{1} \varphi^{\prime}+\psi_{2}^{\prime} \in H_{\mu}^{\infty}, \psi_{2} \varphi^{\prime} \in H_{\mu}^{\infty}$, and

(d) $\lim _{|\varphi(z)| \rightarrow 1} \frac{\mu(z)\left|\psi_{1}(z) \varphi^{\prime}(z)+\psi_{2}^{\prime}(z)\right|}{\left(1-|\varphi(z)|^{2}\right)^{\frac{2+q}{p}}}=0$ and

(e) $\lim _{|\varphi(z)| \rightarrow 1} \frac{\mu(z)\left|\psi_{2}(z) \varphi^{\prime}(z)\right|}{\left(1-|\varphi(z)|^{2}\right)^{\frac{2+q}{p}+1}}=0$.

(iii) If $p=2+q$ and $s>1$, then $T_{\psi_{1}, \psi_{2}, \varphi}^{0}: F(p, q, s)\left(\right.$ or $\left.F_{0}(p, q, s)\right) \rightarrow \mathcal{B}_{\mu}$ is compact if and only if $\psi_{1} \in \mathcal{B}, \psi_{1} \varphi^{\prime}+\psi_{2}^{\prime} \in H_{\mu}^{\infty}, \psi_{2} \varphi^{\prime} \in H_{\mu}^{\infty}$, and

(f) $\lim _{|\varphi(z)| \rightarrow 1} \mu(z)\left|\psi_{1}^{\prime}(z)\right| \ln \left(\frac{2}{1-|\varphi(z)|^{2}}\right)=0$,

(g) $\lim _{|\varphi(z)| \rightarrow 1} \frac{\mu(z)\left|\psi_{1}(z) \varphi^{\prime}(z)+\psi_{2}^{\prime}(z)\right|}{1-|\varphi(z)|^{2}}=0$, and

(h) $\lim _{|\varphi(z)| \rightarrow 1} \frac{\mu(z)\left|\psi_{2}(z) \varphi^{\prime}(z)\right|}{\left(1-|\varphi(z)|^{2}\right)^{2}}=0$.

Proof (i) Suppose that $n \in \mathbb{N}$, or $n=0$ and $p<2+q$, and $T_{\psi_{1}, \psi_{2}, \varphi}^{n}: F(p, q, s)\left(\right.$ or $\left.F_{0}(p, q, s)\right) \rightarrow$ $\mathcal{B}_{\mu}$ is compact. Let $\left(z_{k}\right)_{k \in \mathbb{N}}$ be a sequence in $\mathbb{D}$ such that $\left|\varphi\left(z_{k}\right)\right| \rightarrow 1$ as $k \rightarrow \infty$ (if such a 
sequence does not exist, then (a)-(c) obviously hold). Let

$$
\begin{aligned}
f_{k}(z)= & \prod_{j=1}^{n}\left(\frac{2+q}{p}+j\right)\left(\frac{2+q}{p}+j+1\right) \frac{1-\left|\varphi\left(z_{k}\right)\right|^{2}}{\left(1-\overline{\varphi\left(z_{k}\right)} z\right)^{\frac{2+q}{p}}} \\
& -2 \prod_{j=1}^{n}\left(\frac{2+q}{p}+j-1\right)\left(\frac{2+q}{p}+j+1\right) \frac{\left(1-\left|\varphi\left(z_{k}\right)\right|^{2}\right)^{2}}{\left(1-\overline{\varphi\left(z_{k}\right)} z\right)^{\frac{2+q}{p}+1}} \\
& +\prod_{j=1}^{n}\left(\frac{2+q}{p}+j-1\right)\left(\frac{2+q}{p}+j\right) \frac{\left(1-\left|\varphi\left(z_{k}\right)\right|^{3}\right)^{3}}{\left(1-\overline{\varphi\left(z_{k}\right)} z\right)^{\frac{2+q}{p}+2}}, \\
g_{k}(z)= & \left(\frac{2+q}{p}+n+2\right) \prod_{j=1}^{n}\left(\frac{2+q}{p}+j\right)\left(\frac{2+q}{p}+j+1\right) \frac{1-\left|\varphi\left(z_{k}\right)\right|^{2}}{\left(1-\overline{\left.\varphi\left(z_{k}\right) z\right)^{\frac{2+q}{p}}}\right.} \\
& -\left(2 \frac{(2+q)}{p}+2 n+3\right) \prod_{j=1}^{n}\left(\frac{2+q}{p}+j-1\right)\left(\frac{2+q}{p}+j+1\right) \frac{\left(1-\left|\varphi\left(z_{k}\right)\right|^{2}\right)^{2}}{\left(1-\overline{\varphi\left(z_{k}\right)} z\right)^{\frac{2+q}{p}+1}} \\
& +\left(\frac{2+q}{p}+n+1\right) \prod_{j=1}^{n}\left(\frac{2+q}{p}+j-1\right)\left(\frac{2+q}{p}+j\right) \frac{\left(1-\left|\varphi\left(z_{k}\right)\right|^{3}\right)^{3}}{\left(1-\overline{\left.\varphi\left(z_{k}\right) z\right)^{\frac{2+q}{p}}+2}\right.},
\end{aligned}
$$

and

$$
\begin{aligned}
h_{k}(z)= & \left(\frac{2+q}{p}+n+1\right)\left(\frac{2+q}{p}+n+2\right) \\
& \times \prod_{j=1}^{n}\left(\frac{2+q}{p}+j\right)\left(\frac{2+q}{p}+j+1\right) \frac{1-\left|\varphi\left(z_{k}\right)\right|^{2}}{\left(1-\overline{\varphi\left(z_{k}\right)} z\right)^{\frac{2+q}{p}}} \\
& -2\left(\frac{2+q}{p}+n\right)\left(\frac{2+q}{p}+n+2\right) \\
& \times \prod_{j=1}^{n}\left(\frac{2+q}{p}+j-1\right)\left(\frac{2+q}{p}+j+1\right) \frac{\left(1-\left|\varphi\left(z_{k}\right)\right|^{2}\right)^{2}}{\left(1-\overline{\varphi\left(z_{k}\right)} z\right)^{\frac{2+q}{p}+1}} \\
& +\left(\frac{2+q}{p}+n\right)\left(\frac{2+q}{p}+n+1\right) \\
& \times \prod_{j=1}^{n}\left(\frac{2+q}{p}+j-1\right)\left(\frac{2+q}{p}+j\right) \frac{\left(1-\left|\varphi\left(z_{k}\right)\right|^{3}\right)^{3}}{\left(1-\overline{\varphi\left(z_{k}\right)} z\right)^{\frac{2+q}{p}+2}} .
\end{aligned}
$$

From the proof of Theorem 1 we know that $\left(f_{k}\right)_{k \in \mathbb{N}},\left(g_{k}\right)_{k \in \mathbb{N}}$, and $\left(h_{k}\right)_{k \in \mathbb{N}}$ are norm bounded sequences in $F_{0}(p, q, s)$, and it is easy to see that they converge to zero uniformly on compact subsets of $\mathbb{D}$ as $k \rightarrow \infty$. Hence, by Lemma 5 we have

$$
\begin{aligned}
& \lim _{k \rightarrow \infty}\left\|T_{\psi_{1}, \psi_{2}, \varphi}^{n} f_{k}\right\|_{\mathcal{B}_{\mu}}=0, \quad \lim _{k \rightarrow \infty}\left\|T_{\psi_{1}, \psi_{2}, \varphi}^{n} g_{k}\right\|_{\mathcal{B}_{\mu}}, \quad \text { and } \\
& \lim _{k \rightarrow \infty}\left\|T_{\psi_{1}, \psi_{2}, \varphi}^{n} h_{k}\right\|_{\mathcal{B}_{\mu}}=0 .
\end{aligned}
$$

From (16), (18), and (20) it follows that

$$
\mu(\zeta) \frac{\left|\psi_{2}(\zeta) \varphi^{\prime}(\zeta)(\varphi(\zeta))^{n+2}\right|}{\left(1-|\varphi(\zeta)|^{2}\right)^{\frac{2+q}{p}+n+1}} \lesssim\left\|T_{\psi_{1}, \psi_{2}, \varphi}^{n} f_{k}\right\|_{\mathcal{B}_{\mu}}
$$




$$
\mu(\zeta) \frac{\left|\left(\psi_{1}(\zeta) \varphi^{\prime}(\zeta)+\psi_{2}^{\prime}(\zeta)\right)(\varphi(\zeta))^{n+1}\right|}{\left(1-|\varphi(\zeta)|^{2}\right)^{\frac{2+q}{p}+n}} \lesssim\left\|T_{\psi_{1}, \psi_{2}, \varphi}^{n} g_{k}\right\|_{\mathcal{B}_{\mu}}
$$

and

$$
\mu(\zeta) \frac{\left|\psi_{1}^{\prime}(\zeta)(\varphi(\zeta))^{n}\right|}{\left(1-|\varphi(\zeta)|^{2}\right)^{\frac{2+q}{p}+n-1}} \lesssim\left\|T_{\psi_{1}, \psi_{2}, \varphi}^{n} h_{k}\right\|_{\mathcal{B}_{\mu}}
$$

Letting $k \rightarrow \infty$ in (32)-(34) and employing (31), we get (a), (b), and (c).

Conversely, assume that $\psi_{1} \in \mathcal{B}_{\mu}, \psi_{1} \varphi^{\prime}+\psi_{2}^{\prime} \in H_{\mu}^{\infty}, \psi_{2} \varphi^{\prime} \in H_{\mu}^{\infty}$, and that (a), (b), and (c) hold. Let $\left(f_{k}\right)_{k \in \mathbb{N}}$ be a sequence in $F(p, q, s)$ (or $\left.F_{0}(p, q, s)\right)$ such that $f_{k} \rightarrow 0$ uniformly on compact subsets of $\mathbb{D}$ and $\left\|f_{k}\right\|_{F(p, q, s)} \lesssim 1$. Then $f_{k}^{(n)}, f_{k}^{(n+1)}$, and $f_{k}^{(n+2)}$ converge to zero uniformly on compact subsets of $\mathbb{D}$ as $k \rightarrow \infty$.

Since (a), (b), and (c) hold, for every $\varepsilon>0$, there exists $\delta \in(0,1)$ such that

$$
\frac{\mu(z)\left|\psi_{1}^{\prime}(z)\right|}{\left(1-|\varphi(z)|^{2}\right)^{\frac{2+q}{p}+n-1}}<\varepsilon, \quad \frac{\mu(z)\left|\psi_{1}(z) \varphi^{\prime}(z)+\psi_{2}^{\prime}(z)\right|}{\left(1-|\varphi(z)|^{2}\right)^{\frac{2+q}{p}+n}}<\varepsilon,
$$

and

$$
\frac{\mu(z)\left|\psi_{2}(z) \varphi^{\prime}(z)\right|}{\left(1-|\varphi(z)|^{2}\right)^{\frac{2+q}{p}+n+1}}<\varepsilon
$$

when $\delta<|\varphi(z)|<1$.

Since $\psi_{1} \in \mathcal{B}_{\mu}, \psi_{1} \varphi^{\prime}+\psi_{2}^{\prime} \in H_{\mu}^{\infty}$, and $\psi_{2} \varphi^{\prime} \in H_{\mu}^{\infty}$, we have $N_{1}=\sup _{z \in \mathbb{D}} \mu(z)\left|\psi_{1}^{\prime}(z)\right|<\infty$, $N_{2}=\sup _{z \in \mathbb{D}} \mu(z)\left|\psi_{1}(z) \varphi^{\prime}(z)+\psi_{2}^{\prime}(z)\right|<\infty$, and $N_{3}=\sup _{z \in \mathbb{D}} \mu(z)\left|\psi_{2}(z) \varphi^{\prime}(z)\right|<\infty$. This, together with Lemma 3, yields

$$
\begin{aligned}
\| & T_{\psi_{1}, \psi_{2}, \varphi}^{n} f_{k} \|_{\mathcal{B}_{\mu}} \\
\leq & \sup _{z \in \mathbb{D}} \mu(z)\left|\left(T_{\psi_{1}, \psi_{2}, \varphi}^{n} f_{k}\right)^{\prime}(z)\right| \\
\leq & \sup _{z \in \mathbb{D}} \mu(z)\left|\psi_{1}^{\prime}(z) f_{k}^{(n)}(\varphi(z))\right|+\sup _{z \in \mathbb{D}} \mu(z)\left|\left(\psi_{1}(z) \varphi^{\prime}(z)+\psi_{2}^{\prime}(z)\right) f_{k}^{(n+1)}(\varphi(z))\right| \\
& +\sup _{z \in \mathbb{D}} \mu(z)\left|\psi_{2}(z) \varphi^{\prime}(z) f_{k}^{(n+2)}(\varphi(z))\right| \\
\leq & \sup _{|\varphi(z)| \leq \delta} \mu(z)\left|\psi_{1}^{\prime}(z)\right|\left|f_{k}^{(n)}(\varphi(z))\right|+\sup _{|\varphi(z)|>\delta} \mu(z)\left|\psi_{1}^{\prime}(z)\right|\left|f_{k}^{(n)}(\varphi(z))\right| \\
& +\sup _{|\varphi(z)| \leq \delta} \mu(z)\left|\left(\psi_{1}(z) \varphi^{\prime}(z)+\psi_{2}^{\prime}(z)\right)\right|\left|f_{k}^{(n+1)}(\varphi(z))\right| \\
& +\sup _{|\varphi(z)|>\delta} \mu(z)\left|\left(\psi_{1}(z) \varphi^{\prime}(z)+\psi_{2}^{\prime}(z)\right)\right|\left|f_{k}^{(n+1)}(\varphi(z))\right| \\
& +\sup _{|\varphi(z)| \leq \delta} \mu(z)\left|\psi_{2}(z) \varphi^{\prime}(z)\right|\left|f_{k}^{(n+2)}(\varphi(z))\right|+\sup _{|\varphi(z)|>\delta} \mu(z)\left|\psi_{2}(z) \varphi^{\prime}(z)\right|\left|f_{k}^{(n+2)}(\varphi(z))\right| \\
\lesssim & N_{1} \sup _{|\varphi(z)| \leq \delta}\left|f_{k}^{(n)}(\varphi(z))\right|+\sup _{|\varphi(z)|>\delta} \frac{\mu(z)\left|\psi_{1}^{\prime}(z)\right|}{\left(1-|\varphi(z)|^{2}\right)^{\frac{2+q}{p}+n-1}} \\
& +N_{2} \sup _{|\varphi(z)| \leq \delta}\left|f_{k}^{(n+1)}(\varphi(z))\right|+\sup _{|\varphi(z)|>\delta} \frac{\mu(z)\left|\psi_{1}(z) \varphi^{\prime}(z)+\psi_{2}^{\prime}(z)\right|}{\left(1-|\varphi(z)|^{2}\right)^{\frac{2+q}{p}+n}}
\end{aligned}
$$




$$
\begin{aligned}
& +N_{3} \sup _{|\varphi(z)| \leq \delta}\left|f_{k}^{(n+2)}(\varphi(z))\right|+\sup _{|\varphi(z)|>\delta} \frac{\mu(z)\left|\psi_{2}(z) \varphi^{\prime}(z)\right|}{\left(1-|\varphi(z)|^{2}\right)^{\frac{2+q}{p}+n+1}} \\
\leq & N_{1} \sup _{|\zeta| \leq \delta}\left|f_{k}^{(n)}(\zeta)\right|+N_{2} \sup _{|\zeta| \leq \delta}\left|f_{k}^{(n+1)}(\zeta)\right|+N_{3} \sup _{|\zeta| \leq \delta}\left|f_{k}^{(n+2)}(\zeta)\right|+3 \varepsilon .
\end{aligned}
$$

From the arbitrariness of $\varepsilon$ and the fact that $f_{k}^{(n)}, f_{k}^{(n+1)}$, and $f_{k}^{(n+2)}$ converges to zero uniformly on compact subsets of $\mathbb{D}$ we have $\lim _{k \rightarrow \infty}\left\|T_{\psi_{1}, \psi_{2}, \varphi}^{n} f_{k}\right\|_{\mathcal{B}_{\mu}}=0$, and so by Lemma 5 , $T_{\psi_{1}, \psi_{2}, \varphi}^{n}: F(p, q, s)\left(\right.$ or $\left.F_{0}(p, q, s)\right) \rightarrow \mathcal{B}_{\mu}$ is compact.

(ii) If $T_{\psi_{1}, \psi_{2}, \varphi}^{0}: F(p, q, s)\left(\right.$ or $\left.F_{0}(p, q, s)\right) \rightarrow \mathcal{B}_{\mu}$ is compact, then it is bounded and from Theorem 1 we see that $\psi_{1} \in \mathcal{B}_{\mu}, \psi_{1} \varphi^{\prime}+\psi_{2}^{\prime} \in H_{\mu}^{\infty}$, and $\psi_{2} \varphi^{\prime} \in H_{\mu}^{\infty}$. Relations (d) and (e) follow from the proof of (i) with $n=0$.

Now assume that $p>2+q, \psi_{1} \in \mathcal{B}_{\mu}, \psi_{2} \varphi^{\prime} \in H_{\mu}^{\infty}, \psi_{1} \varphi^{\prime}+\psi_{2}^{\prime} \in H_{\mu}^{\infty}$, and that (d) and (e) hold. Let $\left(f_{k}\right)_{k \in \mathbb{N}}$ be a bounded sequence in $F(p, q, s)$ (or $\left.F_{0}(p, q, s)\right)$ that converges to zero uniformly on compact subsets of $\mathbb{D}$ as $k \rightarrow \infty$. To show that $T_{\psi_{1}, \psi_{2}, \varphi}^{0}$ : $F(p, q, s)\left(\right.$ or $\left.F_{0}(p, q, s)\right) \rightarrow \mathcal{B}_{\mu}$ is compact, we need to show that, for each such sequence, $\left\|T_{\psi_{1}, \psi_{2}, \varphi}^{0} f_{k}\right\|_{\mathcal{B}_{\mu}} \rightarrow 0$ as $k \rightarrow \infty$.

From (d) and (e) we have that for every $\varepsilon>0$, there exists $\delta \in(0,1)$ such that

$$
\frac{\mu(z)\left|\psi_{1}(z) \varphi^{\prime}(z)+\psi_{2}^{\prime}(z)\right|}{\left(1-|\varphi(z)|^{2}\right)^{\frac{2+q}{p}}}<\varepsilon \quad \text { and } \quad \frac{\mu(z)\left|\psi_{2}(z) \varphi^{\prime}(z)\right|}{\left(1-|\varphi(z)|^{2}\right)^{\frac{2+q}{p}+1}}<\varepsilon
$$

when $\delta<|\varphi(z)|<1$.

We have

$$
\begin{aligned}
&\left\|T_{\psi_{1}, \psi_{2}, \varphi}^{0} f_{k}\right\|_{\mathcal{B}_{\mu}} \\
& \leq \sup _{z \in \mathbb{D}} \mu(z)\left|\left(T_{\psi_{1}, \psi_{2}, \varphi}^{0} f_{k}\right)^{\prime}(z)\right| \\
& \leq \sup _{z \in \mathbb{D}} \mu(z)\left|\psi_{1}^{\prime}(z) f_{k}(\varphi(z))\right|+\sup _{z \in \mathbb{D}} \mu(z)\left|\left(\psi_{1}(z) \varphi^{\prime}(z)+\psi_{2}^{\prime}(z)\right) f_{k}^{\prime}(\varphi(z))\right| \\
& \quad+\sup _{z \in \mathbb{D}} \mu(z)\left|\psi_{2}(z) \varphi^{\prime}(z) f_{k}^{\prime \prime}(\varphi(z))\right| \\
& \leq N_{1} \sup _{z \in \mathbb{D}}\left|f_{k}(\varphi(z))\right|+\sup _{|\varphi(z)| \leq \delta} \mu(z)\left|\left(\psi_{1}(z) \varphi^{\prime}(z)+\psi_{2}^{\prime}(z)\right)\right|\left|f_{k}^{\prime}(\varphi(z))\right| \\
& \quad+\sup _{|\varphi(z)|>\delta} \mu(z)\left|\left(\psi_{1}(z) \varphi^{\prime}(z)+\psi_{2}^{\prime}(z)\right)\right|\left|f_{k}^{\prime}(\varphi(z))\right| \\
& \quad+\sup _{|\varphi(z)| \leq \delta} \mu(z)\left|\psi_{2}(z) \varphi^{\prime}(z)\right|\left|f_{k}^{\prime \prime}(\varphi(z))\right|+\sup _{|\varphi(z)|>\delta} \mu(z)\left|\psi_{2}(z) \varphi^{\prime}(z)\right|\left|f_{k}^{\prime \prime}(\varphi(z))\right| \\
& \lesssim N_{1} \sup _{z \in \mathbb{D}}\left|f_{k}(z)\right|+N_{2} \sup _{|\varphi(z)| \leq \delta}\left|f_{k}^{\prime}(\varphi(z))\right|+\sup _{|\varphi(z)|>\delta} \frac{\mu(z)\left|\psi_{1}(z) \varphi^{\prime}(z)+\psi_{2}^{\prime}(z)\right|}{\left(1-|\varphi(z)|^{2}\right)^{\frac{2+q}{p}}} \\
&+N_{3} \sup _{|\varphi(z)| \leq \delta}\left|f_{k}^{\prime \prime}(\varphi(z))\right|+\sup _{|\varphi(z)|>\delta} \frac{\mu(z)\left|\psi_{2}(z) \varphi^{\prime}(z)\right|}{\left(1-|\varphi(z)|^{2}\right)^{\frac{2+q}{p}+1}} \\
& \lesssim N_{1} \sup _{z \in \mathbb{D}}\left|f_{k}(z)\right|+N_{2} \sup _{|\zeta| \leq \delta}\left|f_{k}^{\prime}(\zeta)\right|+N_{3} \sup _{|\zeta| \leq \delta}^{\prime}\left|f_{k}^{\prime}(\zeta)\right|+2 \varepsilon,
\end{aligned}
$$

where $N_{j}, j=\overline{1,3}$, are as in (i).

From this and from the fact that $f_{k}^{\prime}$ and $f_{k}^{\prime \prime}$ converge to zero on compacts of $\mathbb{D}$ as $k \rightarrow \infty$, by Lemma 5.2 in [39] we have $\sup _{z \in \mathbb{D}}\left|f_{k}(z)\right| \rightarrow 0$ as $k \rightarrow \infty$, and by the arbi- 
trariness of $\varepsilon$ it follows that $\lim _{k \rightarrow \infty}\left\|T_{\psi_{1}, \psi_{2}, \varphi}^{0} f_{k}\right\|_{\mathcal{B}_{\mu}}=0$. Hence, by Lemma 5, $T_{\psi_{1}, \psi_{2}, \varphi}^{0}$ : $F(p, q, s)\left(\right.$ or $\left.F_{0}(p, q, s)\right) \rightarrow \mathcal{B}_{\mu}$ is compact.

(iii) If $T_{\psi_{1}, \psi_{2}, \varphi}^{0}: F(p, q, s)\left(\right.$ or $\left.F_{0}(p, q, s)\right) \rightarrow \mathcal{B}_{\mu}$ is compact, then it is bounded, and from Theorem 1 we see that $\psi_{1} \in \mathcal{B}_{\mu}, \psi_{1} \varphi^{\prime}+\psi_{2}^{\prime} \in H_{\mu}^{\infty}$, and $\psi_{2} \varphi^{\prime} \in H_{\mu}^{\infty}$. Relations (g) and (h) follow from the proof in (i) with $n=0$ and $p=2+q$.

Assume that $\left(z_{k}\right)_{k \in \mathbb{N}}$ is a sequence in $\mathbb{D}$ for which $\left|\varphi\left(z_{k}\right)\right| \rightarrow 1$ as $k \rightarrow \infty$ (if such a sequence does not exist, then (f), (g), and (h) obviously hold). Consider the sequence of functions

$$
g_{k}(z)=\frac{20}{\mu_{k}^{2}}\left(\ln \frac{2}{1-\overline{\varphi\left(z_{k}\right) z}}\right)^{3}-\frac{30}{\mu_{k}^{3}}\left(\ln \frac{2}{1-\overline{\varphi\left(z_{k}\right)} z}\right)^{4}+\frac{12}{\mu_{k}^{4}}\left(\ln \frac{2}{1-\overline{\varphi\left(z_{k}\right) z}}\right)^{5}
$$

where

$$
\mu_{k}=\ln \frac{2}{1-\left|\varphi\left(z_{k}\right)\right|^{2}}, \quad k \in \mathbb{N}
$$

Then

$$
\begin{aligned}
g_{k}^{\prime}(z)= & \left(\frac{60}{\mu_{k}^{2}}\left(\ln \frac{2}{1-\overline{\varphi\left(z_{k}\right)} z}\right)^{2}-\frac{120}{\mu_{k}^{3}}\left(\ln \frac{2}{1-\overline{\varphi\left(z_{k}\right)}}\right)^{3}\right. \\
& \left.+\frac{60}{\mu_{k}^{4}}\left(\ln \frac{2}{1-\overline{\varphi\left(z_{k}\right) z}}\right)^{4}\right) \frac{\overline{\varphi\left(z_{k}\right)}}{1-\overline{\varphi\left(z_{k}\right) z}} \\
g_{k}^{\prime \prime}(z)= & \left(\frac{60}{\mu_{k}^{2}}\left(\ln \frac{2}{1-\overline{\varphi\left(z_{k}\right) z}}\right)^{2}-\frac{120}{\mu_{k}^{3}}\left(\ln \frac{2}{1-\overline{\varphi\left(z_{k}\right) z}}\right)^{3}\right. \\
& \left.+\frac{60}{\mu_{k}^{4}}\left(\ln \frac{2}{1-\overline{\varphi\left(z_{k}\right)} z}\right)^{4}\right) \frac{\left(\overline{\varphi\left(z_{k}\right)}\right)^{2}}{\left(1-\overline{\varphi\left(z_{k}\right)} z\right)^{2}} \\
& +\left(\frac{120}{\left(\mu_{k}\right)^{2}}\left(\ln \frac{2}{1-\overline{\varphi\left(z_{k}\right)} z}\right)-\frac{360}{\mu_{k}^{3}}\left(\ln \frac{2}{1-\overline{\varphi\left(z_{k}\right)} z}\right)^{2}\right. \\
& \left.+\frac{240}{\mu_{k}^{4}}\left(\ln \frac{2}{1-\overline{\varphi\left(z_{k}\right) z}}\right)^{3}\right) \frac{\left(\overline{\varphi\left(z_{k}\right)}\right)^{2}}{\left(1-\overline{\left.\varphi\left(z_{k}\right) z\right)^{2}} .\right.}
\end{aligned}
$$

By some calculation we have $g_{k} \in \mathcal{B}_{0}, k \in \mathbb{N}$, and $\sup _{k \in \mathbb{N}}\left\|g_{k}\right\|_{\mathcal{B}} \leq C<\infty$. Since $s>1$, we have $g_{k} \in F_{0}(p, q, s)$ and $\sup _{k \in \mathbb{N}}\left\|g_{k}\right\|_{F(p, q, s)}<\infty$. It is easy to see that $g_{k}$ converges to zero uniformly on compact subsets of $\mathbb{D}$ and that

$$
g_{k}^{\prime}\left(\varphi\left(z_{k}\right)\right)=0, \quad g_{k}^{\prime \prime}\left(\varphi\left(z_{k}\right)\right)=0, \quad \text { and } \quad g_{k}\left(\varphi\left(z_{k}\right)\right)=2 \ln \left(\frac{2}{1-\left|\varphi\left(z_{k}\right)\right|^{2}}\right)
$$

So if $T_{\psi_{1}, \psi_{2}, \varphi}^{0}: F(p, q, s)\left(\right.$ or $\left.F_{0}(p, q, s)\right) \rightarrow \mathcal{B}_{\mu}$ is compact, then $\left\|T_{\psi_{1}, \psi_{2}, \varphi}^{0} g_{k}\right\|_{\mathcal{B}_{\mu}} \rightarrow 0$ as $k \rightarrow$ $\infty$, and, consequently,

$$
\mu\left(z_{k}\right)\left|\psi_{1}^{\prime}\left(z_{k}\right)\right| \ln \left(\frac{2}{1-\left|\varphi\left(z_{k}\right)\right|^{2}}\right) \lesssim\left\|T_{\psi_{1}, \psi_{2}, \varphi}^{0} g_{k}\right\|_{\mathcal{B}_{\mu}} \rightarrow 0
$$

as $k \rightarrow \infty$, so (f) holds. 
Now assume that $p=2+q, \psi_{1} \in \mathcal{B}_{\mu}, \psi_{2} \varphi^{\prime} \in H_{\mu}^{\infty}, \psi_{1} \varphi^{\prime}+\psi_{2}^{\prime} \in H_{\mu}^{\infty}$, and that (f), (g), and (h) hold. Let $\left(f_{k}\right)_{k \in \mathbb{N}}$ be a bounded sequence in $F(p, q, s)$ (or $\left.F_{0}(p, q, s)\right)$ that converges to zero uniformly on compact subsets of $\mathbb{D}$ as $k \rightarrow \infty$.

From (f), (g), and (h) we have that for every $\varepsilon>0$, there exists $\delta \in(0,1)$ such that

$$
\begin{aligned}
& \mu(z)\left|\psi_{1}^{\prime}(z)\right| \ln \left(\frac{2}{1-|\varphi(z)|^{2}}\right)<\varepsilon, \\
& \frac{\mu(z)\left|\psi_{1}(z) \varphi^{\prime}(z)+\psi_{2}^{\prime}(z)\right|}{1-|\varphi(z)|^{2}}<\varepsilon, \quad \text { and } \quad \frac{\mu(z)\left|\psi_{2}(z) \varphi^{\prime}(z)\right|}{\left(1-|\varphi(z)|^{2}\right)^{2}}<\varepsilon
\end{aligned}
$$

when $\delta<|\varphi(z)|<1$.

Using the inequalities, we have

$$
\begin{aligned}
& \left\|T_{\psi_{1}, \psi_{2}, \varphi}^{0} f_{k}\right\|_{\mathcal{B}_{\mu}} \\
& \leq \sup _{z \in \mathbb{D}} \mu(z)\left|\left(T_{\psi_{1}, \psi_{2}, \varphi}^{0} f_{k}\right)^{\prime}(z)\right| \\
& \leq \sup _{z \in \mathbb{D}} \mu(z)\left|\psi_{1}^{\prime}(z) f_{k}(\varphi(z))\right|+\sup _{z \in \mathbb{D}} \mu(z)\left|\left(\psi_{1}(z) \varphi^{\prime}(z)+\psi_{2}^{\prime}(z)\right) f_{k}^{\prime}(\varphi(z))\right| \\
& +\sup _{z \in \mathbb{D}} \mu(z)\left|\psi_{2}(z) \varphi^{\prime}(z) f_{k}^{\prime \prime}(\varphi(z))\right| \\
& \leq \sup _{|\varphi(z)| \leq \delta} \mu(z)\left|\psi_{1}^{\prime}(z) f_{k}(\varphi(z))\right|+\sup _{|\varphi(z)|>\delta} \mu(z)\left|\psi_{1}^{\prime}(z) f_{k}(\varphi(z))\right| \\
& +\sup _{|\varphi(z)| \leq \delta} \mu(z)\left|\left(\psi_{1}(z) \varphi^{\prime}(z)+\psi_{2}^{\prime}(z)\right)\right|\left|f_{k}^{\prime}(\varphi(z))\right| \\
& +\sup _{|\varphi(z)|>\delta} \mu(z)\left|\left(\psi_{1}(z) \varphi^{\prime}(z)+\psi_{2}^{\prime}(z)\right)\right|\left|f_{k}^{\prime}(\varphi(z))\right| \\
& +\sup _{|\varphi(z)| \leq \delta} \mu(z)\left|\psi_{2}(z) \varphi^{\prime}(z)\right|\left|f_{k}^{\prime \prime}(\varphi(z))\right|+\sup _{|\varphi(z)|>\delta} \mu(z)\left|\psi_{2}(z) \varphi^{\prime}(z)\right|\left|f_{k}^{\prime \prime}(\varphi(z))\right| \\
& \lesssim N_{1} \sup _{|\varphi(z)| \leq \delta}\left|f_{k}(\varphi(z))\right|+\sup _{|\varphi(z)|>\delta} \mu(z)\left|\psi_{1}^{\prime}(z)\right| \ln \frac{2}{1-|\varphi(z)|^{2}} \\
& +N_{2} \sup _{|\varphi(z)| \leq \delta}\left|f_{k}^{\prime}(\varphi(z))\right|+\sup _{|\varphi(z)|>\delta} \frac{\mu(z)\left|\psi_{1}(z) \varphi^{\prime}(z)+\psi_{2}^{\prime}(z)\right|}{1-|\varphi(z)|^{2}} \\
& +N_{3} \sup _{|\varphi(z)| \leq \delta}\left|f_{k}^{\prime \prime}(\varphi(z))\right|+\sup _{|\varphi(z)|>\delta} \frac{\mu(z)\left|\psi_{2}(z) \varphi^{\prime}(z)\right|}{\left(1-|\varphi(z)|^{2}\right)^{2}} \\
& \leq N_{1} \sup _{\zeta \in \mathbb{D}}\left|f_{k}(\zeta)\right|+N_{2} \sup _{|\zeta| \leq \delta}\left|f_{k}^{\prime}(\zeta)\right|+N_{3} \sup _{|\zeta| \leq \delta}\left|f_{k}^{\prime \prime}(\zeta)\right|+3 \varepsilon,
\end{aligned}
$$

where $N_{j}, j=\overline{1,3}$, are as in (i).

From this, since $f_{k}, f_{k}^{\prime}$, and $f_{k}^{\prime \prime}$ converge to zero on compacts of $\mathbb{D}$ as $k \rightarrow \infty$, by the arbitrariness of $\varepsilon$ it follows that $\lim _{k \rightarrow \infty}\left\|T_{\psi_{1}, \psi_{2}, \varphi}^{0} f_{k}\right\|_{\mathcal{B}_{\mu}}=0$. Hence, by Lemma $5, T_{\psi_{1}, \psi_{2}, \varphi}^{0}$ : $F(p, q, s)\left(\right.$ or $\left.F_{0}(p, q, s)\right) \rightarrow \mathcal{B}_{\mu}$ is compact.

Theorem 3 Let $0<p, s<\infty,-2<q<\infty, q+s>-1, \psi_{1}, \psi_{2} \in H(\mathbb{D}), n \in \mathbb{N}_{0}, \mu$ be a typical weight, and $\varphi \in S(\mathbb{D})$. Then the following statements are true.

(i) If $n \in \mathbb{N}$, or $n=0$ and $p<2+q$, then $T_{\psi_{1}, \psi_{2}, \varphi}^{n}: F_{0}(p, q, s) \rightarrow \mathcal{B}_{\mu, 0}$ is bounded if and only if $\psi_{1} \in \mathcal{B}_{\mu, 0}, \psi_{1} \varphi^{\prime}+\psi_{2}^{\prime} \in H_{\mu, 0}^{\infty}, \psi_{2} \varphi^{\prime} \in H_{\mu, 0}^{\infty}$, and conditions (a)-(c) of Theorem 1 hold. 
(ii) If $p>2+q$, then $T_{\psi_{1}, \psi_{2}, \varphi}^{0}: F_{0}(p, q, s) \rightarrow \mathcal{B}_{\mu, 0}$ is bounded if and only if $\psi_{1} \in \mathcal{B}_{\mu, 0}$, $\psi_{1} \varphi^{\prime}+\psi_{2}^{\prime} \in H_{\mu, 0}^{\infty}, \psi_{2} \varphi^{\prime} \in H_{\mu, 0}^{\infty}$, and conditions (d) and (e) of Theorem 1 hold.

(iii) If $p=2+q$ and $s>1$, then $T_{\psi_{1}, \psi_{2}, \varphi}^{0}: F_{0}(p, q, s) \rightarrow \mathcal{B}_{\mu, 0}$ is bounded if and only if $\psi_{1} \in \mathcal{B}_{\mu, 0}, \psi_{1} \varphi^{\prime}+\psi_{2}^{\prime} \in H_{\mu, 0}^{\infty}, \psi_{2} \varphi^{\prime} \in H_{\mu, 0}^{\infty}$, and conditions (f)-(h) of Theorem 1 hold.

Proof (i) First, assume that $T_{\psi_{1}, \psi_{2}, \varphi}^{n}: F_{0}(p, q, s) \rightarrow \mathcal{B}_{\mu, 0}$ is bounded. Taking the test functions $p_{k}(z)=z^{k} / k$ ! for $k \in\{n, n+1, n+2\}$, we easily get that $\psi_{1} \in \mathcal{B}_{\mu, 0}$ and that the functions $\psi_{1} \varphi^{\prime}+\psi_{2}^{\prime}$ and $\psi_{2} \varphi^{\prime}$ are in $H_{\mu, 0}^{\infty}$. Moreover, as in the proof of Theorem 1, we can easily show that conditions (a)-(c) hold.

Conversely, suppose that $\psi_{1} \in \mathcal{B}_{\mu, 0}, \psi_{1} \varphi^{\prime}+\psi_{2}^{\prime} \in H_{\mu, 0}^{\infty}, \psi_{2} \varphi^{\prime} \in H_{\mu, 0}^{\infty}$, and that conditions (a)-(c) of Theorem 1 hold. Then by Theorem 1(i) we know that the operator $T_{\psi_{1}, \psi_{2}, \varphi}^{n}$ : $F_{0}(p, q, s) \rightarrow \mathcal{B}_{\mu}$ is bounded. Hence, to show that the operator $T_{\psi_{1}, \psi_{2}, \varphi}^{n}: F_{0}(p, q, s) \rightarrow \mathcal{B}_{\mu, 0}$ is bounded, it suffices to show that $T_{\psi_{1}, \psi_{2}, \varphi}^{n} f \in \mathcal{B}_{\mu, 0}$ for every $f \in F_{0}(p, q, s)$. Take any $\varepsilon>0$. Let $f \in F_{0}(p, q, s)$. Then by Lemma $1, f \in \mathcal{B}_{0}^{(q+2) / p}$. From this by using Propositions 7 and 8 in [2] we have that there is $\delta_{1} \in(0,1)$ such that, for any $z \in \mathbb{D}$ such that $|z|>\delta_{1}$,

$$
\left|f^{(j)}(z)\right|<\frac{\varepsilon}{\left(1-|z|^{2}\right)^{\frac{2+q}{p}+j-1}}
$$

for $j \in\{n, n+1, n+2\}$, where $n \in \mathbb{N}$ or $n=0$ and $p<2+q$.

From this, using conditions (a)-(c) of Theorem 1, respectively, we have that

$$
\begin{aligned}
& \mu(z)\left|\psi_{1}^{\prime}(z) f^{(n)}(\varphi(z))\right|<\varepsilon \frac{\mu(z)\left|\psi_{1}^{\prime}(z)\right|}{\left(1-|\varphi(z)|^{2}\right)^{\frac{2+q}{p}+n-1}} \leq M_{1} \varepsilon \\
& \mu(z)\left|\psi_{1}(z) \varphi^{\prime}(z)+\psi_{2}^{\prime}(z)\right|\left|f^{(n+1)}(\varphi(z))\right|<\varepsilon \frac{\mu(z)\left|\psi_{1}(z) \varphi^{\prime}(z)+\psi_{2}^{\prime}(z)\right|}{\left(1-|\varphi(z)|^{2}\right)^{\frac{2+q}{p}+n}} \leq M_{2} \varepsilon,
\end{aligned}
$$

and

$$
\mu(z)\left|\psi_{2}(z) \varphi^{\prime}(z)\right|\left|f^{(n+2)}(\varphi(z))\right|<\varepsilon \frac{\mu(z)\left|\psi_{2}(z) \varphi^{\prime}(z)\right|}{\left(1-|\varphi(z)|^{2}\right)^{\frac{2+q}{p}+n+1}} \leq M_{3} \varepsilon
$$

when $|\varphi(z)|>\delta_{1}$, where

$$
\begin{aligned}
& M_{1}:=\sup _{|\varphi(z)|>\delta_{1}} \frac{\mu(z)\left|\psi_{1}^{\prime}(z)\right|}{\left(1-|\varphi(z)|^{2}\right)^{\frac{2+q}{p}+n-1}}, \quad M_{2}:=\sup _{|\varphi(z)|>\delta_{1}} \frac{\mu(z)\left|\psi_{1}(z) \varphi^{\prime}(z)+\psi_{2}^{\prime}(z)\right|}{\left(1-|\varphi(z)|^{2}\right)^{\frac{2+q}{p}}+n}, \\
& M_{3}:=\sup _{|\varphi(z)|>\delta_{1}} \frac{\mu(z)\left|\psi_{2}(z) \varphi^{\prime}(z)\right|}{\left(1-|\varphi(z)|^{2}\right)^{\frac{2+q}{p}+n+1}}
\end{aligned}
$$

(note that these quantities are finite due to conditions (a)-(c)).

On the other hand, since $\psi_{1} \in \mathcal{B}_{\mu, 0}, \psi_{1} \varphi^{\prime}+\psi_{2}^{\prime} \in H_{\mu, 0}^{\infty}$, and $\psi_{2} \varphi^{\prime} \in H_{\mu, 0}^{\infty}$, we have that there is $\delta_{2} \in(0,1)$ such that

$$
\begin{aligned}
& \mu(z)\left|\psi_{1}^{\prime}(z)\right|<\varepsilon\left(1-\delta_{1}^{2}\right)^{\frac{2+q}{p}+n-1}, \\
& \mu(z)\left|\psi_{1}(z) \varphi^{\prime}(z)+\psi_{2}^{\prime}(z)\right|<\varepsilon\left(1-\delta_{1}^{2}\right)^{\frac{2+q}{p}+n},
\end{aligned}
$$


and

$$
\mu(z)\left|\psi_{2}(z) \varphi^{\prime}(z)\right|<\varepsilon\left(1-\delta_{1}^{2}\right)^{\frac{2+q}{p}+n+1}
$$

whenever $|z|>\delta_{2}$.

From this, by Lemma 3 we have that, for $|\varphi(z)| \leq \delta_{1}$ and $|z|>\delta_{2}$,

$$
\begin{aligned}
\mu(z)\left|\psi_{1}^{\prime}(z) f^{(n)}(\varphi(z))\right| \lesssim\|f\|_{F(p, q, s)} \frac{\mu(z)\left|\psi_{1}^{\prime}(z)\right|}{\left(1-\delta_{1}^{2}\right)^{\frac{2+q}{p}+n-1}} & \lesssim \varepsilon\|f\|_{F(p, q, s)}, \\
\mu(z)\left|\psi_{1}(z) \varphi^{\prime}(z)+\psi_{2}^{\prime}(z)\right|\left|f^{(n+1)}(\varphi(z))\right| & \lesssim\|f\|_{F(p, q, s)} \frac{\mu(z)\left|\psi_{1}(z) \varphi^{\prime}(z)+\psi_{2}^{\prime}(z)\right|}{\left(1-\delta_{1}^{2}\right)^{\frac{2+q}{p}+n}} \\
& \lesssim \varepsilon\|f\|_{F(p, q, s)},
\end{aligned}
$$

and

$$
\mu(z)\left|\psi_{2}(z) \varphi^{\prime}(z)\right|\left|f^{(n+2)}(\varphi(z))\right| \lesssim\|f\|_{F(p, q, s)} \frac{\mu(z)\left|\psi_{2}(z) \varphi^{\prime}(z)\right|}{\left(1-\delta_{1}^{2}\right)^{\frac{2+q}{p}+n+1}} \lesssim \varepsilon\|f\|_{F(p, q, s)} .
$$

Combining (36) and (39), (37) and (40), and (38) and (41), respectively, we have that, whenever $|z|>\delta_{2}$,

$$
\begin{aligned}
& \mu(z)\left|\psi_{1}^{\prime}(z) f^{(n)}(\varphi(z))\right| \lesssim\left\{M_{1},\|f\|_{F(p, q, s)}\right\} \varepsilon, \\
& \mu(z)\left|\psi_{1}(z) \varphi^{\prime}(z)+\psi_{2}^{\prime}(z)\right|\left|f^{(n+1)}(\varphi(z))\right| \lesssim\left\{M_{2},\|f\|_{F(p, q, s)}\right\} \varepsilon
\end{aligned}
$$

and

$$
\mu(z)\left|\psi_{2}(z) \varphi^{\prime}(z)\right|\left|f^{(n+2)}(\varphi(z))\right| \lesssim\left\{M_{3},\|f\|_{F(p, q, s)}\right\} \varepsilon
$$

Hence,

$$
\begin{aligned}
\mu(z)\left|\left(T_{\psi_{1}, \psi_{2}, \varphi}^{n} f\right)^{\prime}(z)\right| \leq & \mu(z)\left|\psi_{1}^{\prime}(z) f^{(n)}(\varphi(z))\right|+\mu(z)\left|\psi_{1}(z) \varphi^{\prime}(z)+\psi_{2}^{\prime}(z)\right|\left|f^{(n+1)}(\varphi(z))\right| \\
& +\mu(z)\left|\psi_{2}(z) \varphi^{\prime}(z)\right|\left|f^{(n+2)}(\varphi(z))\right| \\
\lesssim & \left\{M_{1}, M_{2}, M_{3},\|f\|_{F(p, q, s)}\right\} \varepsilon,
\end{aligned}
$$

from which it follows that $T_{\psi_{1}, \psi_{2}, \varphi}^{n} f \in \mathcal{B}_{\mu, 0}$ for every $f \in F_{0}(p, q, s)$ and, consequently, the boundedness of the operator $T_{\psi_{1}, \psi_{2}, \varphi}^{n}: F_{0}(p, q, s) \rightarrow \mathcal{B}_{\mu, 0}$.

(ii) If $T_{\psi_{1}, \psi_{2}, \varphi}^{0}: F_{0}(p, q, s) \rightarrow \mathcal{B}_{\mu, 0}$ is bounded, then as in the proof of (i), we get that $\psi_{1} \in$ $\mathcal{B}_{\mu, 0}, \psi_{1} \varphi^{\prime}+\psi_{2}^{\prime} \in H_{\mu, 0}^{\infty}, \psi_{2} \varphi^{\prime} \in H_{\mu, 0}^{\infty}$, and that conditions (d) and (e) of Theorem 1 hold.

Conversely, suppose that $\psi_{1} \in \mathcal{B}_{\mu, 0}, \psi_{1} \varphi^{\prime}+\psi_{2}^{\prime} \in H_{\mu, 0}^{\infty}, \psi_{2} \varphi^{\prime} \in H_{\mu, 0}^{\infty}$, and that conditions (d) and (e) of Theorem 1 hold. Then by Theorem 1(ii) we know that the operator $T_{\psi_{1}, \psi_{2}, \varphi}^{0}: F_{0}(p, q, s) \rightarrow \mathcal{B}_{\mu}$ is bounded. Hence, as in (i), to show that the operator $T_{\psi_{1}, \psi_{2}, \varphi}^{0}: F_{0}(p, q, s) \rightarrow \mathcal{B}_{\mu, 0}$ is bounded, it suffices to show that $T_{\psi_{1}, \psi_{2}, \varphi}^{0} f \in \mathcal{B}_{\mu, 0}$ for every $f \in F_{0}(p, q, s)$. 
Take any $\varepsilon>0$. Then $\psi_{1} \in \mathcal{B}_{\mu, 0}$ implies that there is some $\delta_{1} \in(0,1)$ such that

$$
\mu(z)\left|\psi_{1}(z)\right|<\varepsilon
$$

for $|z|>\delta_{1}$. Let $f \in F_{0}(p, q, s)$. Then by Lemma $1, f \in \mathcal{B}_{0}^{(q+2) / p}$. From this, by Proposition 8 in [2] it follows that there is $\delta_{2} \in(0,1)$ such that, for any $z \in \mathbb{D}$ such that $|z|>\delta_{2}$,

$$
\left|f^{(j)}(z)\right|<\frac{\varepsilon}{\left(1-|z|^{2}\right)^{\frac{2+q}{p}+j-1}}
$$

for $j \in\{1,2\}$.

Thus, using conditions (d) and (e) of Theorem 1 respectively, we have that

$$
\mu(z)\left|\psi_{1}(z) \varphi^{\prime}(z)+\psi_{2}^{\prime}(z)\right|\left|f^{\prime}(\varphi(z))\right|<\varepsilon \frac{\mu(z)\left|\psi_{1}(z) \varphi^{\prime}(z)+\psi_{2}^{\prime}(z)\right|}{\left(1-|\varphi(z)|^{2}\right)^{\frac{2+q}{p}}} \leq N_{1} \varepsilon
$$

and

$$
\mu(z)\left|\psi_{2}(z) \varphi^{\prime}(z)\right|\left|f^{\prime \prime}(\varphi(z))\right|<\varepsilon \frac{\mu(z)\left|\psi_{2}(z) \varphi^{\prime}(z)\right|}{\left(1-|\varphi(z)|^{2}\right)^{\frac{2+q}{p}+1}} \leq N_{2} \varepsilon
$$

when $|\varphi(z)|>\delta_{2}$, where

$$
N_{1}:=\sup _{|\varphi(z)|>\delta_{2}} \frac{\mu(z)\left|\psi_{1}(z) \varphi^{\prime}(z)+\psi_{2}^{\prime}(z)\right|}{\left(1-|\varphi(z)|^{2}\right)^{\frac{2+q}{p}}} \text { and } N_{2}:=\sup _{|\varphi(z)|>\delta_{2}} \frac{\mu(z)\left|\psi_{2}(z) \varphi^{\prime}(z)\right|}{\left(1-|\varphi(z)|^{2}\right)^{\frac{2+q}{p}+1}}
$$

(note that these quantities are finite due to conditions (d) and (e) of Theorem 1).

On the other hand, since $\psi_{1} \varphi^{\prime}+\psi_{2}^{\prime} \in H_{\mu, 0}^{\infty}$ and $\psi_{2} \varphi^{\prime} \in H_{\mu, 0}^{\infty}$, we have that there is $\delta_{3} \in$ $(0,1)$ such that

$$
\mu(z)\left|\psi_{1}(z) \varphi^{\prime}(z)+\psi_{2}^{\prime}(z)\right|<\varepsilon\left(1-\delta_{2}^{2}\right)^{\frac{2+q}{p}}
$$

and

$$
\mu(z)\left|\psi_{2}(z) \varphi^{\prime}(z)\right|<\varepsilon\left(1-\delta_{2}^{2}\right)^{\frac{2+q}{p}+1}
$$

whenever $|z|>\delta_{3}$.

Thus, for $|\varphi(z)| \leq \delta_{2}$ and $|z|>\delta_{3}$, we have that

$$
\begin{aligned}
\mu(z)\left|\psi_{1}(z) \varphi^{\prime}(z)+\psi_{2}^{\prime}(z)\right|\left|f^{\prime}(\varphi(z))\right| & \lesssim\|f\|_{F(p, q, s)} \frac{\mu(z)\left|\psi_{1}(z) \varphi^{\prime}(z)+\psi_{2}^{\prime}(z)\right|}{\left(1-\delta_{2}^{2}\right)^{\frac{2+q}{p}}} \\
& \lesssim \varepsilon\|f\|_{F(p, q, s)}
\end{aligned}
$$

and

$$
\mu(z)\left|\psi_{2}(z) \varphi^{\prime}(z)\right|\left|f^{\prime \prime}(\varphi(z))\right| \lesssim\|f\|_{F(p, q, s)} \frac{\mu(z)\left|\psi_{2}(z) \varphi^{\prime}(z)\right|}{\left(1-\delta_{2}^{2}\right)^{\frac{2+q}{p}+1}} \lesssim \varepsilon\|f\|_{F(p, q, s)} .
$$


Combining (46) and (48) and, respectively, (47) and (49), we have that, whenever $|z|>\delta_{3}$,

$$
\mu(z)\left|\psi_{1}(z) \varphi^{\prime}(z)+\psi_{2}^{\prime}(z)\right|\left|f^{\prime}(\varphi(z))\right| \lesssim\left\{N_{1},\|f\|_{F(p, q, s)}\right\} \varepsilon
$$

and

$$
\mu(z)\left|\psi_{2}(z) \varphi^{\prime}(z)\right|\left|f^{\prime \prime}(\varphi(z))\right| \lesssim\left\{N_{2},\|f\|_{F(p, q, s)}\right\} \varepsilon
$$

From (45), (50), and (51), by Lemma 3 we have that

$$
\begin{aligned}
\mu(z)\left|\left(T_{\psi_{1}, \psi_{2}, \varphi}^{0} f\right)^{\prime}(z)\right| \leq & \mu(z)\left|\psi_{1}^{\prime}(z) f(\varphi(z))\right|+\mu(z)\left|\psi_{1}(z) \varphi^{\prime}(z)+\psi_{2}^{\prime}(z)\right|\left|f^{\prime}(\varphi(z))\right| \\
& +\mu(z)\left|\psi_{2}(z) \varphi^{\prime}(z)\right|\left|f^{\prime \prime}(\varphi(z))\right| \\
\lesssim & \left\{N_{1}, N_{2},\|f\|_{F(p, q, s)}\right\} \varepsilon
\end{aligned}
$$

when $|z|>\max \left\{\delta_{1}, \delta_{3}\right\}$. Hence, $T_{\psi_{1}, \psi_{2}, \varphi}^{0} f \in \mathcal{B}_{\mu, 0}$ for every $f \in F_{0}(p, q, s)$, from which the boundedness of $T_{\psi_{1}, \psi_{2}, \varphi}^{0}: F_{0}(p, q, s) \rightarrow \mathcal{B}_{\mu, 0}$ follows.

(iii) If $T_{\psi_{1}, \psi_{2}, \varphi}^{0}: F_{0}(p, q, s) \rightarrow \mathcal{B}_{\mu, 0}$ is bounded, then as in the proof of (i), we get that $\psi_{1} \in$ $\mathcal{B}_{\mu, 0}, \psi_{1} \varphi^{\prime}+\psi_{2}^{\prime} \in H_{\mu, 0}^{\infty}, \psi_{2} \varphi^{\prime} \in H_{\mu, 0}^{\infty}$, and that conditions (f), (g), and (h) of Theorem 1 hold.

Conversely, suppose that $\psi_{1} \in \mathcal{B}_{\mu, 0}, \psi_{1} \varphi^{\prime}+\psi_{2}^{\prime} \in H_{\mu, 0}^{\infty}, \psi_{2} \varphi^{\prime} \in H_{\mu, 0}^{\infty}$, and conditions (f), (g), and (h) of Theorem 1 hold. Then by Theorem 1(iii) we know that the operator $T_{\psi_{1}, \psi_{2}, \varphi}^{0}: F_{0}(p, q, s) \rightarrow \mathcal{B}_{\mu}$ is bounded. As in the previous two cases, to show that the operator $T_{\psi_{1}, \psi_{2}, \varphi}^{0}: F_{0}(p, q, s) \rightarrow \mathcal{B}_{\mu, 0}$ is bounded, it suffices to show that $T_{\psi_{1}, \psi_{2}, \varphi}^{0} f \in \mathcal{B}_{\mu, 0}$ for every $f \in F_{0}(p, q, s)$.

Take any $\varepsilon>0$. Let $f \in F_{0}(p, q, s)$. Then by Lemma $1, f \in \mathcal{B}_{0}$. From this, by [2], Proposition 8 , and [8], Lemma 3 , for the case $n=1$ with $f^{\prime}$ replaced by $f$, it easily follows that there is $\delta_{1} \in(0,1)$ such that, for any $z \in \mathbb{D}$ such that $|z|>\delta_{1}$,

$$
|f(z)|<\varepsilon \ln \frac{2}{1-|z|^{2}}
$$

and

$$
\left|f^{(j)}(z)\right|<\frac{\varepsilon}{\left(1-|z|^{2}\right)^{j}}
$$

for $j \in\{1,2\}$.

From this, using conditions (f), (g), and (h) of Theorem 1, respectively, we have that

$$
\begin{aligned}
& \mu(z)\left|\psi_{1}^{\prime}(z) f(\varphi(z))\right|<\varepsilon \mu(z)\left|\psi_{1}^{\prime}(z)\right| \ln \frac{2}{1-|\varphi(z)|^{2}} \leq L_{1} \varepsilon \\
& \mu(z)\left|\psi_{1}(z) \varphi^{\prime}(z)+\psi_{2}^{\prime}(z)\right|\left|f^{\prime}(\varphi(z))\right|<\varepsilon \frac{\mu(z)\left|\psi_{1}(z) \varphi^{\prime}(z)+\psi_{2}^{\prime}(z)\right|}{1-|\varphi(z)|^{2}} \leq L_{2} \varepsilon
\end{aligned}
$$

and

$$
\mu(z)\left|\psi_{2}(z) \varphi^{\prime}(z)\right|\left|f^{\prime \prime}(\varphi(z))\right|<\varepsilon \frac{\mu(z)\left|\psi_{2}(z) \varphi^{\prime}(z)\right|}{\left(1-|\varphi(z)|^{2}\right)^{2}} \leq L_{3} \varepsilon
$$


when $|\varphi(z)|>\delta_{1}$, where

$$
\begin{aligned}
& L_{1}:=\sup _{|\varphi(z)|>\delta_{1}} \mu(z)\left|\psi_{1}^{\prime}(z)\right| \ln \frac{2}{1-|\varphi(z)|^{2}}, \quad L_{2}:=\sup _{|\varphi(z)|>\delta_{1}} \frac{\mu(z)\left|\psi_{1}(z) \varphi^{\prime}(z)+\psi_{2}^{\prime}(z)\right|}{1-|\varphi(z)|^{2}}, \\
& L_{3}:=\sup _{|\varphi(z)|>\delta_{1}} \frac{\mu(z)\left|\psi_{2}(z) \varphi^{\prime}(z)\right|}{\left(1-|\varphi(z)|^{2}\right)^{2}}
\end{aligned}
$$

(note that these quantities are finite due to conditions (f), (g), and (h) of Theorem 1).

On the other hand, since $\psi_{1} \in \mathcal{B}_{\mu, 0}, \psi_{1} \varphi^{\prime}+\psi_{2}^{\prime} \in H_{\mu, 0}^{\infty}$, and $\psi_{2} \varphi^{\prime} \in H_{\mu, 0}^{\infty}$, we have that there is $\delta_{2} \in(0,1)$ such that

$$
\mu(z)\left|\psi_{1}^{\prime}(z)\right|<\varepsilon / \ln \frac{2}{1-\delta_{1}^{2}}, \quad \mu(z)\left|\psi_{1}(z) \varphi^{\prime}(z)+\psi_{2}^{\prime}(z)\right|<\varepsilon\left(1-\delta_{1}^{2}\right),
$$

and

$$
\mu(z)\left|\psi_{2}(z) \varphi^{\prime}(z)\right|<\varepsilon\left(1-\delta_{1}^{2}\right)^{2}
$$

whenever $|z|>\delta_{2}$.

Thus, for $|\varphi(z)| \leq \delta_{1}$ and $|z|>\delta_{2}$, we have that

$$
\begin{aligned}
& \mu(z)\left|\psi_{1}^{\prime}(z)\right||f(\varphi(z))| \lesssim\|f\|_{F(p, q, s)} \mu(z)\left|\psi_{1}^{\prime}(z)\right| \ln \frac{2}{1-\delta_{1}^{2}}<\varepsilon\|f\|_{F(p, q, s)} \\
& \mu(z)\left|\psi_{1}(z) \varphi^{\prime}(z)+\psi_{2}^{\prime}(z)\right|\left|f^{\prime}(\varphi(z))\right| \lesssim\|f\|_{F(p, q, s)} \frac{\mu(z)\left|\psi_{1}(z) \varphi^{\prime}(z)+\psi_{2}^{\prime}(z)\right|}{1-\delta_{1}^{2}} \\
&<\varepsilon\|f\|_{F(p, q, s)},
\end{aligned}
$$

and

$$
\mu(z)\left|\psi_{2}(z) \varphi^{\prime}(z)\right|\left|f^{\prime \prime}(\varphi(z))\right| \lesssim\|f\|_{F(p, q, s)} \frac{\mu(z)\left|\psi_{2}(z) \varphi^{\prime}(z)\right|}{\left(1-\delta_{1}^{2}\right)^{2}}<\varepsilon\|f\|_{F(p, q, s)} .
$$

Combining (52) and (55), (53) and (56), and (54) and (57), respectively, we have that, whenever $|z|>\delta_{2}$,

$$
\begin{aligned}
& \mu(z)\left|\psi_{1}^{\prime}(z)\right||f(\varphi(z))| \lesssim\left\{L_{1},\|f\|_{F(p, q, s)}\right\} \varepsilon, \\
& \mu(z)\left|\psi_{1}(z) \varphi^{\prime}(z)+\psi_{2}^{\prime}(z)\right|\left|f^{\prime}(\varphi(z))\right| \lesssim\left\{L_{2},\|f\|_{F(p, q, s)}\right\} \varepsilon,
\end{aligned}
$$

and

$$
\mu(z)\left|\psi_{2}(z) \varphi^{\prime}(z)\right|\left|f^{\prime \prime}(\varphi(z))\right| \lesssim\left\{L_{3},\|f\|_{F(p, q, s)}\right\} \varepsilon
$$

Thus, from (58), (59), and (60) we have that

$$
\begin{aligned}
\mu(z)\left|\left(T_{\psi_{1}, \psi_{2}, \varphi}^{0} f\right)^{\prime}(z)\right| \leq & \mu(z)\left|\psi_{1}^{\prime}(z) f(\varphi(z))\right|+\mu(z)\left|\psi_{1}(z) \varphi^{\prime}(z)+\psi_{2}(z)\right|\left|f^{\prime}(\varphi(z))\right| \\
& +\mu(z)\left|\psi_{2}(z) \varphi^{\prime}(z)\right|\left|f^{\prime \prime}(\varphi(z))\right| \\
\lesssim & \left\{L_{1}, L_{2}, L_{3},\|f\|_{F(p, q, s)}\right\} \varepsilon
\end{aligned}
$$


when $|z|>\delta_{2}$. Hence, $T_{\psi_{1}, \psi_{2}, \varphi}^{0} f \in \mathcal{B}_{\mu, 0}$ for every $f \in F_{0}(p, q, s)$, from which the boundedness of $T_{\psi_{1}, \psi_{2}, \varphi}^{0}: F_{0}(p, q, s) \rightarrow \mathcal{B}_{\mu, 0}$ follows.

Theorem 4 Let $0<p, s<\infty,-2<q<\infty, q+s>-1, \psi_{1}, \psi_{2} \in H(\mathbb{D}), n \in \mathbb{N}_{0}$, $\mu$ be a typical weight, and $\varphi \in S(\mathbb{D})$.

(i) If $n \in \mathbb{N}$, or $n=0$ and $p<2+q$, and $T_{\psi_{1}, \psi_{2}, \varphi}^{n}: F(p, q, s) \rightarrow \mathcal{B}_{\mu, 0}$ is bounded, then $\psi_{1} \in$ $\mathcal{B}_{\mu, 0}, \psi_{1} \varphi^{\prime}+\psi_{2}^{\prime} \in H_{\mu, 0}^{\infty}, \psi_{2} \varphi^{\prime} \in H_{\mu, 0}^{\infty}$, and conditions (a)-(c) of Theorem 1 hold.

Also, if $\psi_{1} \in \mathcal{B}_{\mu, 0}, \psi_{1} \varphi^{\prime}+\psi_{2}^{\prime} \in H_{\mu, 0}^{\infty}, \psi_{2} \varphi^{\prime} \in H_{\mu, 0}^{\infty}$, and conditions (a)-(c) of Theorem 2 hold, then $T_{\psi_{1}, \psi_{2}, \varphi}^{n}: F(p, q, s) \rightarrow \mathcal{B}_{\mu, 0}$ is bounded.

(ii) If $2+q<p$ and $T_{\psi_{1}, \psi_{2}, \varphi}^{0}: F(p, q, s) \rightarrow \mathcal{B}_{\mu, 0}$ is bounded, then $\psi_{1} \in \mathcal{B}_{\mu, 0}, \psi_{1} \varphi^{\prime}+\psi_{2}^{\prime} \in H_{\mu, 0}^{\infty}$, $\psi_{2} \varphi^{\prime} \in H_{\mu, 0}^{\infty}$, and conditions (d) and (f) of Theorem 1 hold.

Also, if $\psi_{1} \in \mathcal{B}_{\mu, 0}, \psi_{1} \varphi^{\prime}+\psi_{2}^{\prime} \in H_{\mu, 0}^{\infty}, \psi_{2} \varphi^{\prime} \in H_{\mu, 0}^{\infty}$, and conditions (d) and (f) of Theorem 2 hold, then $T_{\psi_{1}, \psi_{2}, \varphi}^{0}: F(p, q, s) \rightarrow \mathcal{B}_{\mu, 0}$ is bounded.

(iii) If $2+q=p, s>1$, and $T_{\psi_{1}, \psi_{2}, \varphi}^{0}: F(p, q, s) \rightarrow \mathcal{B}_{\mu, 0}$ is bounded, then $\psi_{1} \in \mathcal{B}_{\mu, 0}, \psi_{1} \varphi^{\prime}+$ $\psi_{2}^{\prime} \in H_{\mu, 0}^{\infty}, \psi_{2} \varphi^{\prime} \in H_{\mu, 0}^{\infty}$, and conditions (f)-(h) of Theorem 1 hold.

Also, if $\psi_{1} \in \mathcal{B}_{\mu, 0}, \psi_{1} \varphi^{\prime}+\psi_{2}^{\prime} \in H_{\mu, 0}^{\infty}, \psi_{2} \varphi^{\prime} \in H_{\mu, 0}^{\infty}$, and conditions (f)-(h) of Theorem 2 hold, then $T_{\psi_{1}, \psi_{2}, \varphi}^{0}: F(p, q, s) \rightarrow \mathcal{B}_{\mu, 0}$ is bounded.

Proof (i) Suppose that $n \in \mathbb{N}$, or $n=0$ and $p<2+q$, and that $T_{\psi_{1}, \psi_{2}, \varphi}^{n}: F(p, q, s) \rightarrow \mathcal{B}_{\mu, 0}$ is bounded. Then $T_{\psi_{1}, \psi_{2}, \varphi}^{n}: F_{0}(p, q, s) \rightarrow \mathcal{B}_{\mu, 0}$ is also bounded, and so by (i) of Theorem 3, $\psi_{1} \in \mathcal{B}_{\mu, 0}, \psi_{1} \varphi^{\prime}+\psi_{2}^{\prime} \in H_{\mu, 0}^{\infty}, \psi_{2} \varphi^{\prime} \in H_{\mu, 0}^{\infty}$, and conditions (a)-(c) of Theorem 1 hold.

Conversely, suppose that $\psi_{1} \in \mathcal{B}_{\mu, 0}, \psi_{1} \varphi^{\prime}+\psi_{2}^{\prime} \in H_{\mu, 0}^{\infty}, \psi_{2} \varphi^{\prime} \in H_{\mu, 0}^{\infty}$, and conditions (a)-(c) of Theorem 2 hold. Then by Theorem 2(i) we have that $T_{\psi_{1}, \psi_{2}, \varphi}^{n}: F(p, q, s) \rightarrow \mathcal{B}_{\mu}$ is compact. Hence, to show the boundedness of $T_{\psi_{1}, \psi_{2}, \varphi}^{n}: F(p, q, s) \rightarrow \mathcal{B}_{\mu, 0}$, it suffices to show that $T_{\psi_{1}, \psi_{2}, \varphi}^{n} f \in \mathcal{B}_{\mu, 0}$ for every $f \in F(p, q, s)$. Take any $\varepsilon>0$. By conditions (a)-(c) of Theorem 2 there is $\delta_{1} \in(0,1)$ such that

$$
\begin{aligned}
& \frac{\mu(z)\left|\psi_{1}^{\prime}(z)\right|}{\left(1-|\varphi(z)|^{2}\right)^{\frac{2+q}{p}+n-1}}<\varepsilon, \\
& \frac{\mu(z)\left|\psi_{1}(z) \varphi^{\prime}(z)+\psi_{2}^{\prime}(z)\right|}{\left(1-|\varphi(z)|^{2}\right)^{\frac{2+q}{p}+n}}<\varepsilon,
\end{aligned}
$$

and

$$
\frac{\mu(z)\left|\psi_{2}(z) \varphi^{\prime}(z)\right|}{\left(1-|\varphi(z)|^{2}\right)^{\frac{2+q}{p}+n+1}}<\varepsilon
$$

when $|\varphi(z)|>\delta_{1}$.

On the other hand, since $\psi_{1} \in \mathcal{B}_{\mu, 0}, \psi_{1} \varphi^{\prime}+\psi_{2}^{\prime} \in H_{\mu, 0}^{\infty}$, and $\psi_{2} \varphi^{\prime} \in H_{\mu, 0}^{\infty}$, we have that there is $\delta_{2} \in(0,1)$ such that

$$
\mu(z)\left|\psi_{1}^{\prime}(z)\right|<\varepsilon\left(1-\delta_{1}^{2}\right)^{\frac{2+q}{p}+n-1}, \quad \mu(z)\left|\psi_{1}(z) \varphi^{\prime}(z)+\psi_{2}^{\prime}(z)\right|<\varepsilon\left(1-\delta_{1}^{2}\right)^{\frac{2+q}{p}+n},
$$

and

$$
\mu(z)\left|\psi_{2}(z) \varphi^{\prime}(z)\right|<\varepsilon\left(1-\delta_{1}^{2}\right)^{\frac{2+q}{p}+n+1}
$$

whenever $|z|>\delta_{2}$. 
Thus, for $|\varphi(z)| \leq \delta_{1}$ and $|z|>\delta_{2}$, we have that

$$
\begin{aligned}
& \frac{\mu(z)\left|\psi_{1}^{\prime}(z)\right|}{\left(1-|\varphi(z)|^{2}\right)^{\frac{2+q}{p}+n-1}} \leq \frac{\mu(z)\left|\psi_{1}^{\prime}(z)\right|}{\left(1-\delta_{1}^{2}\right)^{\frac{2+q}{p}+n-1}}<\varepsilon \\
& \frac{\mu(z)\left|\psi_{1}(z) \varphi^{\prime}(z)+\psi_{2}^{\prime}(z)\right|}{\left(1-|\varphi(z)|^{2}\right)^{\frac{2+q}{p}+n}} \leq \frac{\mu(z)\left|\psi_{1}(z) \varphi^{\prime}(z)+\psi_{2}^{\prime}(z)\right|}{\left(1-\delta_{1}^{2}\right)^{\frac{2+q}{p}+n}}<\varepsilon,
\end{aligned}
$$

and

$$
\frac{\mu(z)\left|\psi_{2}(z) \varphi^{\prime}(z)\right|}{\left(1-|\varphi(z)|^{2}\right)^{\frac{2+q}{p}+n+1}} \leq \frac{\mu(z)\left|\psi_{2}(z) \varphi^{\prime}(z)\right|}{\left(1-\delta_{1}^{2}\right)^{\frac{2+q}{p}+n+1}}<\varepsilon .
$$

Combining (61) and (64), (62) and (65), and (63) and (66), respectively, we have that, whenever $|z|>\delta_{2}$,

$$
\begin{aligned}
\mu(z)\left|\left(T_{\psi_{1}, \psi_{2}, \varphi}^{n} f\right)^{\prime}(z)\right| \leq & \mu(z)\left|\psi_{1}^{\prime}(z) f^{(n)}(\varphi(z))\right|+\mu(z)\left|\psi_{1}(z) \varphi^{\prime}(z)+\psi_{2}(z)\right|\left|f^{(n+1)}(\varphi(z))\right| \\
& +\mu(z)\left|\psi_{2}(z) \varphi^{\prime}(z)\right|\left|f^{(n+2)}(\varphi(z))\right| \\
\lesssim & \|f\|_{F(p, q, s)} \varepsilon
\end{aligned}
$$

from which it follows that $T_{\psi_{1}, \psi_{2}, \varphi}^{n} f \in \mathcal{B}_{\mu, 0}$ for every $f \in F(p, q, s)$, as desired.

(ii) and (iii) These statements are proved similarly to (i). Hence, we omit the details.

The following lemma was essentially proved in [40].

Lemma 6 Let $\mu$ be a typical weight. Then a closed set $K$ in $\mathcal{B}_{\mu, 0}$ is compact if and only if $K$ is bounded and satisfies

$$
\lim _{|z| \rightarrow 1} \sup _{f \in K} \mu(z)\left|f^{\prime}(z)\right|=0 .
$$

Theorem 5 Let $0<p, s<\infty,-2<q<\infty, q+s>-1, \psi_{1}, \psi_{2} \in H(\mathbb{D}), n \in \mathbb{N}_{0}$, $\mu$ be a typical weight, and $\varphi \in S(\mathbb{D})$. Then the following statements hold.

(i) If $n \in \mathbb{N}$, or $n=0$ and $p<2+q$, then $T_{\psi_{1}, \psi_{2}, \varphi}^{n}: F(p, q, s)\left(\right.$ or $\left.F_{0}(p, q, s)\right) \rightarrow \mathcal{B}_{\mu, 0}$ is compact if and only if

(a) $\lim _{|z| \rightarrow 1} \frac{\mu(z)\left|\psi_{1}^{\prime}(z)\right|}{\left(1-|\varphi(z)|^{2}\right)^{\frac{2+q}{p}+n-1}}=0$,

(b) $\lim _{|z| \rightarrow 1} \frac{\mu(z)\left|\psi_{1}(z) \varphi^{\prime}(z)+\psi_{2}^{\prime}(z)\right|}{\left(1-|\varphi(z)|^{2}\right)^{\frac{2+q}{p}+n}}=0$, and

(c) $\lim _{|z| \rightarrow 1} \frac{\mu(z)\left|\psi_{2}(z) \varphi^{\prime}(z)\right|}{\left(1-|\varphi(z)|^{2}\right)^{\frac{2+q}{p}+n+1}}=0$.

(ii) If $p>2+q$, then $T_{\psi_{1}, \psi_{2}, \varphi}^{0}: F(p, q, s)\left(\right.$ or $\left.F_{0}(p, q, s)\right) \rightarrow \mathcal{B}_{\mu, 0}$ is compact if and only if $\psi_{1} \in \mathcal{B}_{\mu, 0}$ and

(d) $\lim _{|z| \rightarrow 1} \frac{\mu(z)\left|\psi_{1}(z) \varphi^{\prime}(z)+\psi_{2}^{\prime}(z)\right|}{\left(1-|\varphi(z)|^{2}\right)^{\frac{2+q}{p}}}=0$ and

(e) $\lim _{|z| \rightarrow 1} \frac{\mu(z)\left|\psi_{2}(z) \varphi^{\prime}(z)\right|}{\left(1-|\varphi(z)|^{2}\right)^{\frac{2+q}{p}+1}}=0$.

(iii) If $p=2+q$ and $s>1$, then $T_{\psi_{1}, \psi_{2}, \varphi}^{0}: F(p, q, s)\left(\right.$ or $\left.F_{0}(p, q, s)\right) \rightarrow \mathcal{B}_{\mu, 0}$ is compact if and only if

(f) $\lim _{|z| \rightarrow 1} \mu(z)\left|\psi_{1}^{\prime}(z)\right| \ln \left(\frac{2}{1-|\varphi(z)|^{2}}\right)=0$, 
(g) $\lim _{|z| \rightarrow 1} \frac{\mu(z)\left|\psi_{1}(z) \varphi^{\prime}(z)+\psi_{2}^{\prime}(z)\right|}{1-|\varphi(z)|^{2}}=0$, and

(h) $\lim _{|z| \rightarrow 1} \frac{\mu(z)\left|\psi_{2}(z) \varphi^{\prime}(z)\right|}{\left(1-|\varphi(z)|^{2}\right)^{2}}=0$.

Proof (i) Suppose that $n \in \mathbb{N}$, or $n=0$ and $p<2+q$, and that (a), (b), and (c) hold. Then by Theorem 1 the operator $T_{\psi_{1}, \psi_{2}, \varphi}^{n}: F(p, q, s)$ (or $\left.F_{0}(p, q, s)\right) \rightarrow \mathcal{B}_{\mu}$ is bounded, $\psi_{1} \in \mathcal{B}_{\mu, 0}, \psi_{1} \varphi^{\prime}+\psi_{2}^{\prime} \in H_{\mu, 0}^{\infty}$, and $\psi_{2} \varphi^{\prime} \in H_{\mu, 0}^{\infty}$, from which it follows that $T_{\psi_{1}, \psi_{2}, \varphi}^{n}$ : $F(p, q, s)$ (or $\left.F_{0}(p, q, s)\right) \rightarrow \mathcal{B}_{\mu, 0}$ is bounded, and, consequently, its image of the unit ball in $F(p, q, s)$.

Hence, due to Lemma 6, to show that $T_{\psi_{1}, \psi_{2}, \varphi}^{n}: F(p, q, s)\left(\right.$ or $\left.F_{0}(p, q, s)\right) \rightarrow \mathcal{B}_{\mu, 0}$ is compact, we only need to show that

$$
\lim _{|z| \rightarrow 1} \sup _{\|f\|_{F(p, q, s)} \leq 1} \mu(z)\left|\left(T_{\psi_{1}, \psi_{2}, \varphi}^{n} f\right)^{\prime}(z)\right|=0 .
$$

From (8) we have

$$
\begin{aligned}
\mu(z)\left|\left(T_{\psi_{1}, \psi_{2}, \varphi}^{n} f\right)^{\prime}(z)\right| \lesssim & \left(\frac{\mu(z)\left|\psi_{1}^{\prime}(z)\right|}{\left(1-|\varphi(z)|^{2}\right)^{\frac{2+q}{p}+n-1}}+\frac{\mu(z)\left|\psi_{1}(z) \varphi^{\prime}(z)+\psi_{2}^{\prime}(z)\right|}{\left(1-|\varphi(z)|^{2}\right)^{\frac{2+q}{p}+n}}\right. \\
& \left.+\frac{\mu(z)\left|\psi_{2}(z) \varphi^{\prime}(z)\right|}{\left(1-|\varphi(z)|^{2}\right)^{\frac{2+q}{p}+n+1}}\right)\|f\|_{F(p, q, s) .}
\end{aligned}
$$

Taking the supremum in (68) over all $f \in F(p, q, s)$ (or $\left.F_{0}(p, q, s)\right)$ such that $\|f\|_{F(p, q, s)} \leq$ 1 , then letting $|z| \rightarrow 1$, and using conditions (a)-(c), we see that (67) holds, so $T_{\psi_{1}, \psi_{2}, \varphi}^{n}$ : $F(p, q, s)\left(\right.$ or $\left.F_{0}(p, q, s)\right) \rightarrow \mathcal{B}_{\mu, 0}$ is compact.

Conversely, suppose that $T_{\psi_{1}, \psi_{2}, \varphi}^{n}: F(p, q, s)\left(\right.$ or $\left.F_{0}(p, q, s)\right) \rightarrow \mathcal{B}_{\mu, 0}$ is compact. Then $T_{\psi_{1}, \psi_{2}, \varphi}^{n}: F(p, q, s)\left(\right.$ or $\left.F_{0}(p, q, s)\right) \rightarrow \mathcal{B}_{\mu}$ is compact, so by Theorem 1 we have

$$
\begin{aligned}
& \lim _{|\varphi(z)| \rightarrow 1} \frac{\mu(z)\left|\psi_{1}^{\prime}(z)\right|}{\left(1-|\varphi(z)|^{2}\right)^{\frac{2+q}{p}+n-1}}=0, \\
& \lim _{|\varphi(z)| \rightarrow 1} \frac{\mu(z)\left|\psi_{1}(z) \varphi^{\prime}(z)+\psi_{2}^{\prime}(z)\right|}{\left(1-|\varphi(z)|^{2}\right)^{\frac{2+q}{p}+n}}=0,
\end{aligned}
$$

and

$$
\lim _{|\varphi(z)| \rightarrow 1} \frac{\mu(z)\left|\psi_{2}(z) \varphi^{\prime}(z)\right|}{\left(1-|\varphi(z)|^{2}\right)^{\frac{2+q}{p}+n+1}}=0 .
$$

On the other hand, using the test functions $f(z)=z^{k} / k !, k \in\{n, n+1, n+2\}$, which belong to $F_{0}(p, q, s)$, we have

$$
\begin{aligned}
& \lim _{|z| \rightarrow 1} \mu(z)\left|\psi_{1}^{\prime}(z)\right|=0, \\
& \lim _{|z| \rightarrow 1} \mu(z)\left|\psi_{1}(z) \varphi^{\prime}(z)+\psi_{2}^{\prime}(z)\right|=0, \\
& \lim _{|z| \rightarrow 1} \mu(z)\left|\psi_{2}(z) \varphi^{\prime}(z)\right|=0 .
\end{aligned}
$$


From (69) we have that for any $\varepsilon>0$, there exists $t \in(0,1)$ such that

$$
\frac{\mu(z)\left|\psi_{1}^{\prime}(z)\right|}{\left(1-|\varphi(z)|^{2}\right)^{\frac{2+q}{p}+n-1}}<\varepsilon
$$

when $t<|\varphi(z)|<1$. From (72) it follows that there exists $r \in(0,1)$ such that

$$
\mu(z)\left|\psi_{1}^{\prime}(z)\right|<\varepsilon\left(1-t^{2}\right)^{\frac{2+q}{p}+n-1}
$$

when $r<|z|<1$.

Therefore, when $r<|z|<1$ and $t<|\varphi(z)|<1$, we have

$$
\frac{\mu(z)\left|\psi_{1}^{\prime}(z)\right|}{\left(1-|\varphi(z)|^{2}\right)^{\frac{2+q}{p}+n-1}}<\varepsilon
$$

whereas when $r<|z|<1$ and $|\varphi(z)| \leq t$, using (76), we have

$$
\frac{\mu(z)\left|\psi_{1}^{\prime}(z)\right|}{\left(1-|\varphi(z)|^{2}\right)^{\frac{2+q}{p}+n-1}}<\varepsilon \frac{\left(1-t^{2}\right)^{\frac{2+q}{p}+n-1}}{\left(1-|\varphi(z)|^{2}\right)^{\frac{2+q}{p}+n-1}} \leq \varepsilon
$$

Since $\varepsilon$ is an arbitrary positive number, we get

$$
\lim _{|z| \rightarrow 1} \frac{\mu(z)\left|\psi_{1}^{\prime}(z)\right|}{\left(1-|\varphi(z)|^{2}\right)^{\frac{2+q}{p}+n-1}}=0
$$

that is, condition (a) holds.

Similarly, we prove that (70) and (73) imply (b) and that (71) and (74) imply (c).

(ii) Assume that $\psi_{1} \in \mathcal{B}_{\mu, 0}$ and that (d) and (e) hold. Then by Theorem 1(ii), $T_{\psi_{1}, \psi_{2}, \varphi}^{0}$ : $F(p, q, s)\left(\right.$ or $\left.F_{0}(p, q, s)\right) \rightarrow \mathcal{B}_{\mu}$ is bounded, $\psi_{1} \varphi^{\prime}+\psi_{2}^{\prime} \in H_{\mu, 0}^{\infty}$, and $\psi_{2} \varphi^{\prime} \in H_{\mu, 0}^{\infty}$. From all these facts it follows that $T_{\psi_{1}, \psi_{2}, \varphi}^{0}: F(p, q, s)\left(\right.$ or $\left.F_{0}(p, q, s)\right) \rightarrow \mathcal{B}_{\mu, 0}$ is bounded, and, consequently, so is its image of the unit ball in $F(p, q, s)$.

Suppose that $f \in F(p, q, s)$ (or $\left.F_{0}(p, q, s)\right)$ is such that $\|f\|_{F(p, q, s)} \leq 1$. Then by Lemma 3 we have

$$
\mu(z)\left|\left(T_{\psi_{1}, \psi_{2}, \varphi}^{0} f\right)^{\prime}(z)\right| \lesssim \mu(z)\left|\psi_{1}^{\prime}(z)\right|+\frac{\mu(z)\left|\psi_{1}(z) \varphi^{\prime}(z)+\psi_{2}^{\prime}(z)\right|}{\left(1-|\varphi(z)|^{2}\right)^{\frac{2+q}{p}}}+\frac{\mu(z)\left|\psi_{2}(z) \varphi^{\prime}(z)\right|}{\left(1-|\varphi(z)|^{2}\right)^{\frac{1+q}{p}+1}}
$$

from which, along with the assumptions $\psi \in \mathcal{B}_{\mu, 0}$, (d), and (e), we have

$$
\lim _{|z| \rightarrow 1} \sup \left\{\mu(z)\left|\left(T_{\psi_{1}, \psi_{2}, \varphi}^{0} f\right)^{\prime}(z)\right|: f \in F(p, q, s)\left(\text { or } F_{0}(p, q, s)\right),\|f\|_{F(p, q, s)} \leq 1\right\}=0
$$

and so by Lemma 6 we get that $T_{\psi_{1}, \psi_{2}, \varphi}^{0}: F(p, q, s)\left(\right.$ or $\left.F_{0}(p, q, s)\right) \rightarrow \mathcal{B}_{\mu, 0}$ is compact.

Conversely, if $T_{\psi_{1}, \psi_{2}, \varphi}^{0}: F(p, q, s)$ (or $\left.F_{0}(p, q, s)\right) \rightarrow \mathcal{B}_{\mu, 0}$ is compact, then for $f(z) \equiv 1$, we obtain that $T_{\psi_{1}, \psi_{2}, \varphi}^{0} 1=\psi_{1} \in \mathcal{B}_{0}$. Relations (d) and (e) follow from the proof of (i) with $n=0$.

(iii) Assume that (f), (g), and (h) hold. Then by Theorem 1(iii) the operator $T_{\psi_{1}, \psi_{2}, \varphi}^{0}$ : $F(p, q, s)\left(\right.$ or $\left.F_{0}(p, q, s)\right) \rightarrow \mathcal{B}_{\mu}$ is bounded, $\psi_{1} \in \mathcal{B}_{\mu, 0}, \psi_{1} \varphi^{\prime}+\psi_{2}^{\prime} \in H_{\mu, 0}^{\infty}$, and $\psi_{2} \varphi^{\prime} \in H_{\mu, 0}^{\infty}$. 
Thus, the operator $T_{\psi_{1}, \psi_{2}, \varphi}^{0}: F(p, q, s)\left(\right.$ or $\left.F_{0}(p, q, s)\right) \rightarrow \mathcal{B}_{\mu, 0}$ is bounded, and so is its image of the unit ball in $F(p, q, s)$.

By Lemma 3 we have that for every $f \in F(p, q, s)\left(\right.$ or $\left.F_{0}(p, q, s)\right)$ such that $\|f\|_{F(p, q, s)} \leq 1$, the following asymptotic relation holds:

$$
\begin{aligned}
\mu(z)\left|\left(T_{\psi_{1}, \psi_{2}, \varphi}^{0} f\right)^{\prime}(z)\right| \lesssim & \mu(z)\left|\psi_{1}^{\prime}(z)\right|\left(\ln \frac{2}{1-|\varphi(z)|^{2}}\right)+\frac{\mu(z)\left|\psi_{1}(z) \varphi^{\prime}(z)+\psi_{2}^{\prime}(z)\right|}{1-|\varphi(z)|^{2}} \\
& +\frac{\mu(z)\left|\psi_{2}(z) \varphi^{\prime}(z)\right|}{\left(1-|\varphi(z)|^{2}\right)^{2}}
\end{aligned}
$$

Taking the supremum in (77) over all $f \in F(p, q, s)$ (or $\left.F_{0}(p, q, s)\right)$ such that $\|f\|_{F(p, q, s)} \leq 1$, then letting $|z| \rightarrow 1$, and using Lemma 6 , we obtain that $T_{\psi_{1}, \psi_{2}, \varphi}^{0}: F(p, q, s)\left(\right.$ or $\left.F_{0}(p, q, s)\right) \rightarrow$ $\mathcal{B}_{\mu, 0}$ is compact.

If $T_{\psi_{1}, \psi_{2}, \varphi}^{0}: F(p, q, s)\left(\right.$ or $\left.F_{0}(p, q, s)\right) \rightarrow \mathcal{B}_{\mu, 0}$ is compact, then $T_{\psi_{1}, \psi_{2}, \varphi}^{0}: F(p, q, s) \quad\left(\right.$ or $F_{0}(p$, $q, s)) \rightarrow \mathcal{B}_{\mu}$ is compact too. Hence, by Theorem 2(iii) we have that

$$
\lim _{|\varphi(z)| \rightarrow 1} \mu(z)\left|\psi_{1}^{\prime}(z)\right| \ln \left(\frac{2}{1-|\varphi(z)|^{2}}\right)=0,
$$

which means that, for every $\varepsilon>0$, there is $t \in(0,1)$ such that

$$
\mu(z)\left|\psi_{1}^{\prime}(z)\right| \ln \frac{2}{1-|\varphi(z)|^{2}}<\varepsilon
$$

when $t<|\varphi(z)|<1$.

On the other hand, $\psi_{1} \in \mathcal{B}_{\mu, 0}$, which implies that there is $r \in(0,1)$ such that

$$
\mu(z)\left|\psi_{1}^{\prime}(z)\right|<\varepsilon / \ln \frac{2}{1-t^{2}}
$$

when $r<|z|<1$. Hence, (78) holds when $r<|z|<1$ and $t<|\varphi(z)|<1$, whereas form (79) we see that (78) holds when $r<|z|<1$ and $|\varphi(z)| \leq t$, so that (f) holds.

Relations (g) and (h) follow from the proof of (i) with $n=0$ and $p=2+q$.

\section{Competing interests}

The authors declare that they have no competing interests.

\section{Authors' contributions}

All authors contributed equally to writing this paper. All authors read and approved the manuscript.

\section{Author details}

'Mathematical Institute of the Serbian Academy of Sciences, Knez Mihailova 36/III, Beograd, 11000, Serbia. ${ }^{2}$ Operator Theory and Applications Research Group, Department of Mathematics, King Abdulaziz University, P.O. Box 80203, Jeddah, 21589, Saudi Arabia. ${ }^{3}$ Department of Mathematics, Shri Mata Vaishno Devi University, Kakryal, Katra, J\&K 182320, India.

\section{Acknowledgements}

Ram Krishan is thankful to DST (India) for Inspire fellowship (DST/Inspire fellowship/2013/281).

Received: 26 May 2016 Accepted: 31 August 2016 Published online: 13 September 2016

\section{References}

1. Avetisyan, K: Hardy-Bloch type spaces and lacunary series on the polydisk. Glasg. Math. J. 49, 345-356 (2007)

2. Zhu, K: Bloch type spaces of analytic functions. Rocky Mt. J. Math. 23, 1143-1177 (1993)

3. Bierstedt, KD, Summers, WH: Biduals of weighted Banach spaces of analytic functions. J. Aust. Math. Soc. Ser. A 54, 70-79 (1993)

4. Zhao, R: On a general family of function space. Ann. Acad. Sci. Fenn., Math. Diss. 105, 1-56 (1996) 
5. Li, H, Guo, Z: On a product-type operator from Zygmund-type spaces to Bloch-Orlicz spaces. J. Inequal. Appl. 2015, Article ID 132 (2015)

6. Li, S, Stević, S: Composition followed by differentiation between Bloch type spaces. J. Comput. Anal. Appl. 9, 195-205 (2007)

7. Li, S, Stević, S: Composition followed by differentiation from mixed-norm spaces to $\alpha$-Bloch spaces. Sb. Math. 199(12), 1847-1857 (2008)

8. Li, S, Stević, S: Cesàro type operators on some spaces of analytic functions on the unit ball. Appl. Math. Comput. 208, 378-388 (2009)

9. Li, S, Stević, S: Composition followed by differentiation between $H^{\infty}$ and $\alpha$-Bloch spaces. Houst. J. Math. 35, 327-340 $(2009)$

10. Li, S, Stević, S: Products of composition and differentiation operators from Zygmund spaces to Bloch spaces and Bers spaces. Appl. Math. Comput. 217, 3144-3154 (2010)

11. Liu, Y, Yu, Y: On a Li-Stević integral-type operator from Bloch-type spaces into logarithmic Bloch spaces. Integral Transforms Spec. Funct. 21(2), 93-103 (2010)

12. Liu, Y, Yu, Y: On a Stević-Sharma operator from Hardy spaces to the logarithmic Bloch spaces. J. Inequal. Appl. 2014, Article ID 22 (2014)

13. Ohno, S: Products of composition and differentiation between Hardy spaces. Bull. Aust. Math. Soc. 73(2), 235-243 (2006)

14. Sharma, AK: Products of multiplication, composition and differentiation between weighted Bergman-Nevanlinna and Bloch-type spaces. Turk. J. Math. 35, 275-291 (2011)

15. Stević, S: Norm and essential norm of composition followed by differentiation from $\alpha$-Bloch spaces to $H_{\mu}^{\infty}$. Appl. Math. Comput. 207, 225-229 (2009)

16. Stević, S: Products of composition and differentiation operators on the weighted Bergman space. Bull. Belg. Math. Soc. Simon Stevin 16, 623-635 (2009)

17. Stević, S: Weighted differentiation composition operators from mixed-norm spaces to weighted-type spaces. Appl. Math. Comput. 211, 222-233 (2009)

18. Stević, S: Weighted differentiation composition operators from mixed-norm spaces to the $n$th weighted-type space on the unit disk. Abstr. Appl. Anal. 2010, Article ID 246287 (2010)

19. Stević, S: Weighted differentiation composition operators from $H^{\infty}$ and Bloch spaces to $n$th weigthed-type spaces on the unit disk. Appl. Math. Comput. 216, 3634-3641 (2010)

20. Stević, S: Characterizations of composition followed by differentiation between Bloch-type spaces. Appl. Math Comput. 218, 4312-4316 (2011)

21. Stević, S: Weighted iterated radial operators between different weighted Bergman spaces on the unit ball. Appl. Math. Comput. 218, 8288-8294 (2012)

22. Stević, S, Sharma, AK: Iterated differentiation followed by composition from Bloch-type spaces to weighted BMOA spaces. Appl. Math. Comput. 218, 3574-3580 (2011)

23. Stević, S, Sharma, AK, Bhat, A: Products of multiplication composition and differentiation operators on weighted Bergman spaces. Appl. Math. Comput. 217, 8115-8125 (2011)

24. Stević, S, Sharma, AK, Bhat, A: Essential norm of products of multiplication composition and differentiation operators on weighted Bergman spaces. Appl. Math. Comput. 218, 2386-2397 (2011)

25. Yang, W: Products of composition and differentiation operators from $\mathcal{Q}_{k}(p, q)$ spaces to Bloch-type spaces. Abstr. Appl. Anal. 2009, Article ID 741920 (2009)

26. Yang, W: Generalized weighted composition operators from the $F(p, q, s)$ space to the Bloch-type space. Appl. Math. Comput. 218, 4967-4972 (2012)

27. Yu, Y, Liu, Y: On Li-Stević integral-type operators between different weighted Bloch-type spaces. J. Inequal. Appl. 2008, Article ID 780845 (2008)

28. Zhou, J, Zhu, X: Product of differentiation and composition operators on the logarithmic Bloch space. J. Inequal. Appl. 2014, Article ID 453 (2014)

29. Zhu, X: Generalized weighted composition operators from Bloch-type spaces to weighted Bergman spaces. Indian J. Math. 49(2), 139-149 (2007)

30. Zhu, X: Products of differentiation, composition and multiplication from Bergman type spaces to Bers spaces. Integral Transforms Spec. Funct. 18(3), 223-231 (2007)

31. Zhu, X: Multiplication followed by differentiation on Bloch-type spaces. Bull. Allahabad Math. Soc. 23(1), 25-39 (2008)

32. Zhu, X: Generalized composition operators from generalized weighted Bergman spaces to Bloch type spaces. J. Korean Math. Soc. 46(6), 1219-1232 (2009)

33. Pan, C: Generalized composition operators from $\mu$-Bloch spaces into mixed norm spaces. Ars Comb. 102, 263-268 (2011)

34. Stević, S: On some integral-type operators between a general space and Bloch-type spaces. Appl. Math. Comput. 218, 2600-2618 (2011)

35. Stević, S, Ueki, SI: Integral-type operators acting between weighted-type spaces on the unit ball. Appl. Math. Comput. 215, 2464-2471 (2009)

36. Zhang, X: Multipliers on some holomorphic function spaces. Chin. Ann. Math., Ser. A 26(4)، 477-486 (2005) (in Chinese)

37. Rudin, W: Function Theory in the Unit Ball of $\mathbb{C}^{n}$. Springer, New York (1980)

38. Schwartz, HJ: Composition operators on $H^{P}$. Thesis, University of Toledo (1969)

39. Ohno, S, Stroethoff, K, Zhao, R: Weighted composition operators between Bloch-type spaces. Rocky Mt. J. Math. 33, $191-215(2003)$

40. Madigan, K, Matheson, A: Compact composition operators on the Bloch space. Trans. Am. Math. Soc. 347, 2679-2687 (1995) 